\title{
Classification of polarized deformation quantizations
}

\author{
Joseph Donin* \\ Dept. of Math., Bar-Ilan University
}

\begin{abstract}
We give a classification of polarized deformation quantizations on a symplectic manifold with a (complex) polarization.

Also, we establish a formula which relates the characteristic class of a polarized deformation quantization to its Fedosov class and the Chern class of the polarization.
\end{abstract}

\section{Contents}

1 Introduction 2

\begin{tabular}{|lll}
\hline 2 & Complex distributions & 6
\end{tabular}

2.1 The Kostant-Hochschild-Rosenberg theorem in presence of a distribution . 7

2.2 Differential operators in presence of a distribution . . . . . . . . . . . . . . . 9

2.3 Differential forms in presence of a distribution . . . . . . . . . . . . . . . . 10

\begin{tabular}{lll}
\hline 3 & C-symplectic manifolds and their polarizations & 11
\end{tabular}

3.1 C-symplectic manifolds . . . . . . . . . . . . . . . . . . . . . . . . . . . . 11

3.2 Polarization . . . . . . . . . . . . . . . . . . . . . . . . . 11

\begin{tabular}{|ll|}
\hline 4 Deformations of a polarized symplectic manifold & 12
\end{tabular}

4.1 Formal everything . . . . . . . . . . . . . . . . . . . . . . . . . . . . . . . . 12

4.2 Local structure of deformed polarizations . . . . . . . . . . . . . . . . . . . 13

4.3 Action of formal automorphisms on a symplectic form . . . . . . . . . . . . 14

*Partially supported by Israel Academy of Sciences Grant no. 8007/99-01 
5 Polarized symplectic connection and characteristic class of a polarized symplectic manifold $\quad 15$

5.1 Polarized symplectic connection . . . . . . . . . . . . . . . . . . . . . 15

5.2 Characteristic class of a polarized symplectic manifold . . . . . . . . . . . 16

\begin{tabular}{lll}
\hline 6 & Deformations of Poisson brackets & 17
\end{tabular}

7 Deformation quantization on a polarized symplectic manifold $\quad 19$

7.1 Deformation quantization . . . . . . . . . . . . . . . . . . . . 19

7.2 Polarized deformation quantization . . . . . . . . . . . . . . . . . . 21

8 Characteristic classes of PDQ's and PSP's 24

8.1 Extension class associated with a PDQ . . . . . . . . . . . . . . . . . . . 24

8.2 Characteristic 2-form associated with a PSP . . . . . . . . . . . . . . . . . 25

8.3 Characteristic pairs for PSP's and PDQ's . . . . . . . . . . . . . . . . . 27

9 Existence of polarized deformation quantizations and relation between the extension and Fedosov classes of a PDQ 28

9.1 Some notations . . . . . . . . . . . . . . . . . . . . . . . . . . . . . 29

9.2 The Fedosov algebra . . . . . . . . . . . . . . . . . . . . . . . . 30

9.3 Lie subalgebras in $\mathcal{W}$. . . . . . . . . . . . . . . . . . . . . 31

9.4 Filtrations on $\mathcal{W}$. . . . . . . . . . . . . . . . . . . . . . . . 32

9.5 Fedosov's construction in the Wick case . . . . . . . . . . . . . . . . . . . . 32

9.6 Existence of PSP's $\ldots \ldots \ldots \ldots$

$\begin{array}{ll}10 \text { The main theorem and corollaries } & 38\end{array}$

\section{Introduction}

Let $(M, \omega)$ be a symplectic manifold, $\mathcal{T}_{M}$ its complexified tangent bundle. It is known that classes of deformation quantizations on $(M, \omega)$ are in one-to-one correspondence with their Fedosov classes, the elements of $\omega+t H^{2}(M, \mathbb{C}[[t]])$. The set $\omega+t H^{2}(M, \mathbb{C}[[t]])$ may be interpreted in the following way. Let $\mathcal{X}$ be the set of formal closed 2-forms on $M$ of the form $\omega+t \omega_{1}+t^{2} \omega_{2}+\cdots$. Let $A u t(M)$ be the group of formal automorphisms of $M$ of the form $e^{t X}$, where $X=X_{0}+t X_{1}+t^{2} X_{2}+\cdots, X_{i} \in \mathcal{T}_{M}$ is a formal vector field. Since 
$\omega$ is nondegenerate, it is easy to see that the orbit of an element $\omega_{t} \in \mathcal{X}$ under the action of $\operatorname{Aut}(M)$ is $\omega_{t}+t \cdot d\left(\Gamma\left(M, \mathcal{T}_{M}^{*}\right)\right)$. It follows from this that $\omega+t H^{2}(M, \mathbb{C}[[t]])$ may be identified with the set of orbits in $\mathcal{X}$ under the $A u t(M)$-action. So, the equivalence classes of deformation quantizations on $(M, \omega)$ are in one-to-one correspondence with the orbits in $\mathcal{X}$.

In the paper, we extend that picture to polarized deformation quantizations (PDQ).

Let $(M, \omega, \mathcal{P})$ be a polarized symplectic manifold, i.e. $\mathcal{P}$ is a Lagrangian integrable subbundle of the complexified tangent bundle to $M$. A PDQ on $(M, \omega, \mathcal{P})$ is a pair $\left(\mathbb{A}_{t}, \mathbb{O}_{t}\right)$, where $\mathbb{A}_{t}$ is a deformation quantization on $(M, \omega)$ and $\mathbb{O}_{t}$ is a commutative $t$ adically complete subalgebra of $\mathbb{A}_{t}$ such that $\mathbb{O}_{0}=\mathcal{O}_{\mathcal{P}}$, the algebra of functions constant along $\mathcal{P}$.

Let $\mathcal{Y}$ denote the set of pairs $\left(\omega_{t}, \mathcal{P}_{t}\right)$, where $\omega_{t} \in \mathcal{X}$ and $\mathcal{P}_{t}$ is a polarization of $\omega_{t}$ such that $\mathcal{P}_{0}=\mathcal{P}$. Our result is that the equivalence classes of PDQ's on $(M, \omega, \mathcal{P})$ are in one-to-one correspondence with the orbits in $\mathcal{Y}$ under the Aut $(M)$-action. Let us describe this correspondence more precisely.

First, we show that any PDQ is equivalent to a polarized star-product (PSP). By a PSP we mean a triple, $\left(\mathcal{C}_{M}^{\infty}[[t]], \mu_{t}, \mathcal{O}_{t}\right)$, where $\left(\mathcal{C}_{M}^{\infty}[[t]], \mu_{t}\right)$ is a star-product, $\mathcal{O}_{t}=\mathcal{O}_{\mathcal{P}_{t}}$, the algebra of functions from $\mathcal{C}_{M}^{\infty}[[t]]$ constant along a deformed polarization $\mathcal{P}_{t}$, and the multiplication $\mu_{t}$ satisfies the condition: $\mu_{t}(f, g)=f g$ (the usual multiplication) for $f \in \mathcal{O}_{t}, g \in \mathcal{C}_{M}^{\infty}[[t]]$.

Further, we assign to any $\operatorname{PSP}\left(\mathcal{C}_{M}^{\infty}[[t]], \mu_{t}, \mathcal{O}_{t}\right)$ a pair $\left(\omega_{t}, \mathcal{P}_{t}\right) \in \mathcal{Y}$ in the following way. We put $\mathcal{P}_{t}=\mathcal{P}_{\mathcal{O}_{t}}$, the sheaf of formal vector fields annihilating $\mathcal{O}_{t}$. The form $\omega_{t}$ is equal, locally, to $\sum_{i} d y_{i} \wedge d x_{i}$, where $x_{i} \in \mathcal{O}_{t}, y_{i} \in \mathcal{C}_{M}^{\infty}[[t]], i=1, \ldots, \frac{1}{2} \operatorname{dim} M$, are Darboux coordinates with respect to $[]=,[,]_{\mu_{t}}$, the commutator of $\mu_{t}$; namely $\left[x_{i}, x_{j}\right]=\left[y_{i}, y_{j}\right]=0$, $\left[y_{i}, x_{j}\right]=\delta_{i j}$. It turns out that $\omega_{t}$ is well defined, i.e. does not depend on the choice of local Darboux coordinates. Denote the constructed map from PSP's to $\mathcal{Y}$ by $\tau$.

The map $\tau$ turns out to descend to an isomorphism between the set of classes of PDQ's and the set $[\mathcal{Y}]$ of orbits in $\mathcal{Y}$. So, we obtain the following commutative diagram of maps:

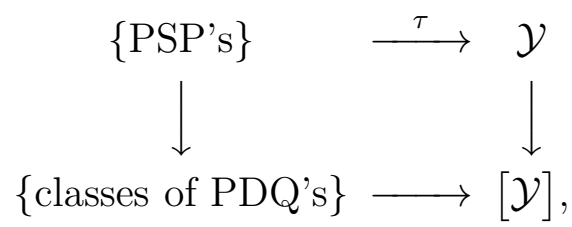

where the left downward arrow is an epimorphism and the bottom arrow is an isomorphism of sets.

We show that the top arrow $\tau$ is an epimorphism with the following properties. 
1) Two PSP's are equivalent if and only if their images with respect to $\tau$ lie on the same orbit.

2) Two PSP's $\left(\mathcal{C}_{M}^{\infty}[[t]], \mu_{t}, \mathcal{O}_{t}\right)$ and $\left(\mathcal{C}_{M}^{\infty}[[t]], \widetilde{\mu}_{t}, \widetilde{\mathcal{O}}_{t}\right)$ have the same image with respect to $\tau$ if and only if $\mathcal{O}_{t}=\widetilde{\mathcal{O}}_{t}$ and $[,]_{\mu_{t}}=[,]_{\widetilde{\mu}_{t}}$.

3) Let $\left(\omega_{t}, \mathcal{P}_{t}\right)=\tau\left(\mathcal{C}_{M}^{\infty}[[t]], \mu_{t}, \mathcal{O}_{t}\right)$. Then, the 2-form

$$
\theta_{t}=\omega_{t}+\frac{t}{2} \operatorname{tr}\left(\left.\nabla^{2}\right|_{\mathcal{P}_{t}}\right)
$$

represents the Fedosov class of the star-product $\left(\mathcal{C}_{M}^{\infty}[[t]], \mu_{t}\right)$. Here $\nabla$ is a connection on $M$ preserving $\omega_{t}, \mathcal{P}_{t}$, and flat on $\mathcal{P}_{t}$ along $\mathcal{P}_{t}$. We prove that such a connection always exists and for it $\operatorname{tr}\left(\left.\nabla^{2}\right|_{\mathcal{P}_{t}}\right)$ belongs to $\Gamma\left(M, d \mathcal{P}_{t}^{\perp}\right)$.

By definition, $\omega_{t}$ belongs to $\omega+t \Gamma\left(M, d \mathcal{P}_{t}^{\perp}\right)$. So, it follows from (1.2) that the Fedosov class of the star-product $\left(\mathcal{C}_{M}^{\infty}[[t]], \mu_{t}\right)$ can be represented by a 2 -form belonging to $\omega+$ $t \Gamma\left(M, d \mathcal{P}_{t}^{\perp}\right)$, as well. In particular, both $\left(M, \omega_{t}, \mathcal{P}_{t}\right)$ and $\left(M, \theta_{t}, \mathcal{P}_{t}\right)$ are formal polarized symplectic manifolds that are deformations of $(M, \omega, \mathcal{P})$.

Another consequence of $(1.2)$ is the following one. Let $\mathbb{A}_{t}$ be a deformation quantization on $(M, \omega)$. Suppose its Fedosov class $c l_{F}\left(\mathbb{A}_{t}\right)$ is represented by the 2 -form $\theta_{t}$ that has a polarization $\mathcal{P}_{t}$. Then $\mathbb{A}_{t}$ can be extended to a $\operatorname{PDQ}\left(\mathbb{A}_{t}, \mathbb{O}_{t}\right)$, i.e. there exists a commutative subalgebra $\mathbb{O}_{t} \subset \mathbb{A}_{t}$ isomorphic to $\mathcal{O}_{\mathcal{P}_{t}}$.

There is the following interpretation of the image $\left[\omega_{t}\right]$ of element $\omega_{t}$ in the $\mathbb{C}[[t]]$-module $\Gamma\left(M, d \mathcal{P}_{t}^{\perp}\right) / d\left(\Gamma\left(M, \mathcal{P}_{t}^{\perp}\right)\right)$. Let $F\left(\mu_{t}, \mathcal{O}_{t}\right)=\left\{a \in \mathcal{C}_{M}^{\infty}[[t]] ;\left[a, \mathcal{O}_{t}\right]_{\mu_{t}} \subset \mathcal{O}_{t}\right\}$. Then, there is the following exact sequence of $\mathcal{O}_{t}$-module and Lie algebra sheaves:

$$
0 \longrightarrow \mathcal{O}_{t} \longrightarrow F\left(\mu_{t}, \mathcal{O}_{t}\right) \longrightarrow \operatorname{Der}\left(\mathcal{O}_{t}\right) \longrightarrow 0
$$

According to [BB] and [BK], $F\left(\mu_{t}, \mathcal{O}_{t}\right)$ is called an $\mathcal{O}_{t}$-extension of $\operatorname{Der}\left(\mathcal{O}_{t}\right)$. Equivalence classes of such extensions are described by their extension classes that are elements of $\Gamma\left(M, d \mathcal{P}_{t}^{\perp}\right) / d\left(\Gamma\left(M, \mathcal{P}_{t}^{\perp}\right)\right)$. We show that $\left[\omega_{t}\right]$ is just the extension class of (1.3).

Analogously, $-\left[\operatorname{tr}\left(\left.\nabla^{2}\right|_{\mathcal{P}_{t}}\right)\right]$ is the extension class of the extension

$$
0 \longrightarrow \mathcal{O}_{t} \longrightarrow \widetilde{T}_{\operatorname{det}\left(\mathcal{P}_{t}\right)} \longrightarrow \operatorname{Der}\left(\mathcal{O}_{t}\right) \longrightarrow 0
$$

where $\widetilde{T}_{\operatorname{det}\left(\mathcal{P}_{t}\right)}$ is the sheaf of $\mathcal{O}_{t}$-differential operators of order at most one on the $\mathcal{O}_{t}$-line bundle $\operatorname{det}\left(\mathcal{P}_{t}\right)$.

Note that $-\operatorname{tr}\left(\left.\nabla^{2}\right|_{\mathcal{P}_{t}}\right)$ divided by $2 \pi \sqrt{-1}$ represents the first Chern class of $\mathcal{P}$, [KN]. So, formula (1.2) gives a relation between the Fedosov and extension classes of a PDQ.

Among results related to ours we mention the following. 
In [RY, N. Reshetikhin and M. Yakimov considered the case of a real polarization on $M$ defined by a Lagrangian fiber bundle $M \rightarrow B$.

In [Ka1], A. Karabegov constructed star-products with separation of variables on Kähler manifolds. This case corresponds to two polarizations on $M$ defined by holomorphic and anti-holomorphic vector fields. In the case of quantization on a Kähler manifold with separation of variables the class of $\omega_{t}$ in $H^{2}(M, \mathbb{C}[[t]])$ coincides with the class defined by Karabegov in [Ka1]. A formula relating the Karabegov and Fedosov classes in case of Kähler manifolds is found in [Ka2], see also [KS], [N]].

Our proof of the existence of a polarized star-product associated with any orbit in $\mathcal{Y}$ uses the Fedosov method adapted for the case with polarization. The analogous method was applied by M. Bordemann and S. Waldmann, BW], for constructing a quantization with separation of variables on a Kähler manifold.

Another approach to proving a formula relating the Fedosov and extension classes, using the Deligne classes, was presented in BD. Unfortunately, there is a deficiency in the proof of Lemma 4.3 of that paper relating the extension and Deligne classes, however, the proof becomes correct for PSP's with the same polarization.

The paper is organized as follows.

In Section 2, we study cohomologies of the differential Hochschild complex on $M$ in presence of a distribution. Also, we prove a version of the Kostant-Hochschild-Rosenberg theorem for functions constant along a distribution. We use these results latter in proving that any PDQ is equivalent to a PSP.

In Section 3, we introduce a notion of $\mathbb{C}$-symplectic manifold, which will be convenient for our consideration. This notion is a generalization of the notion of symplectic manifold. Namely, we suppose that symplectic form $\omega$ on a $\mathbb{C}$-symplectic manifold is a complex one and, locally, there exist complex Darboux coordinates with respect to $\omega$. For a usual symplectic manifold, when $\omega$ is real, such coordinates exist by the Darboux theorem. In this section, we establish some facts on $\mathbb{C}$-symplectic manifolds with polarization. By a polarization of $\omega$ we mean a Lagrangian subbundle, $\mathcal{P}$, of the complexified tangent bundle on $M$ such that, locally on $M$, there exist Darboux coordinates $x_{i}, y_{i}, i=1, \ldots, \frac{1}{2} \operatorname{dim} M$, where $x_{i} \in \mathcal{O}_{\mathcal{P}}$ for all $i$. So, (pseudo-)Kähler manifolds as well as purely real polarizations are included in our considerations. Note that from an analog of "Dolbeault Lemma" proved in $\mathrm{Ra}$ one can derive sufficient conditions for $\mathcal{P}$ to be a complex polarization of $\omega$.

In Section 4, we study properties of formal (or deformed) polarized symplectic manifolds. In Section 5, we prove the existence of a polarized symplectic connection on a 
formal polarized symplectic manifold, $\left(M, \omega_{t}, \mathcal{P}_{t}\right)$, and with the help of it introduce the characteristic class $\widetilde{c}_{1}\left(M, \omega_{t}, \mathcal{P}_{t}\right)$ of a polarized symplectic manifold.

In Section 6, we prove some technical statements related to deformations of Poisson brackets on $M$. Such deformations appear, in particular, as commutators of star-products.

In Section 7, we study properties of PDQ's. In particular, we prove the important fact that any $\mathrm{PDQ}$ is equivalent to a PSP.

In Section 8, we define the extension class of a PDQ. Besides, we assign to any PSP an element of $\mathcal{Y}$, and to any class of PDQ's an orbit in $\mathcal{Y}$. We prove that the later assignment is a monomorphism that, actually, is an isomorphism, as we show in the next section.

In Section 9, we prove that each element of $\mathcal{Y}$ corresponds to a PSP. To this end, we adapt the Fedosov method for constructing a PSP corresponding to a given pair $\left(\omega_{t}, \mathcal{P}_{t}\right) \in$ $\mathcal{Y}$. By this method, a polarized symplectic connection, $\nabla$, extends to a Fedosov connection on the bundle of Weyl algebras on $M$. This connection has two scalar curvatures: the Weyl curvature, $\theta_{t}$, and the Wick curvature that turns out to be just $\omega_{t}$. We show that these curvatures differ from each other by $\frac{t}{2} \operatorname{tr}\left(\left.\nabla^{2}\right|_{\mathcal{P}_{t}}\right)$, which immediately proves (1.2).

In Section 10, we formulate the main theorem collecting the results of the paper and give some corollaries.

Acknowledgments. I thank J.Bernstein, P.Bressler, B.Fedosov, A.Karabegov, and A.Mudrov for helpful discussions.

\section{Complex distributions}

For a smooth manifold $M$ we will denote by $\mathcal{C}_{M}^{\infty}$ the sheaf of complex valued smooth functions on $M$ and by $\mathcal{T}_{M}^{\mathbb{C}}=\mathcal{T}_{M} \otimes_{\mathbb{R}} \mathbb{C}$ the complexified tangent bundle on $M$.

We say that a set of smooth functions $x_{i}, i=1, \ldots, \operatorname{dim} M$, given on an open subset $U \subset M$ form a system of (complex) coordinates on $U$, if $d x_{i}$ are linearly independent at each point of $U$.

Since any 1-form on $U$ can be uniquely written as $\sum_{i} a_{i} d x_{i}$, one can define vector fields $\partial / \partial x_{i} \in \mathcal{T}_{M}^{\mathbb{C}}$ in the following way. If $f$ is a function on $U$ and $d f=\sum_{i} a_{i} d x_{i}$, then $\left(\partial / \partial x_{i}\right) f=a_{i}$.

Let $Q$ be a subbundle in a complex vector bundle $E$ over $M$. We denote by $Q^{\perp}$ the subbundle in $E^{*}$, the complex dual to $E$, orthogonal to $Q$. If sections $e_{i}$ form a local frame in $Q$, we set $\left(e_{i}\right)^{\perp}=Q^{\perp}$.

A (complex) distribution on a manifold $M$ is a subbundle of $\mathcal{T}_{M}^{\mathbb{C}}$. 
Definition 2.1. A distribution $\mathcal{P}$ is said to be integrable if, locally on $M$, there exist (complex valued) functions $f_{1}, \ldots, f_{k}$ such that $d f_{1}, \ldots, d f_{k}$ give a local frame in $\mathcal{P}^{\perp}$, i.e. $d f_{i}$ are linearly independent at each point and $\mathcal{P}=\left(d f_{i}\right)^{\perp}$.

An integrable distribution $\mathcal{P}$ is obviously involutive, i.e. $[\mathcal{P}, \mathcal{P}] \subset \mathcal{P}$.

Let $\mathcal{P}$ be an integrable distribution on $M$. We will denote by $\mathcal{O}_{\mathcal{P}}$ the sheaf of functions on $M$ constant along $\mathcal{P}$, i.e. $f \in \mathcal{O}_{\mathcal{P}}$ if and only if $X f=0$ for any vector field $X \in \mathcal{P}$.

\subsection{The Kostant-Hochschild-Rosenberg theorem in presence of a distribution}

Let $M$ be a smooth manifold. Let $\mathcal{D}^{n}$ be the sheaf of $n$-differential operators on $M$ and $\mathcal{D}^{\bullet}$ the corresponding Hochschild complex with differential $d$. Let $\wedge^{\bullet} \mathcal{T}$ be the complex of sheaves of polyvector fields on $M$ with zero differential, $\mathcal{T}=\mathcal{T}_{M}^{\mathbb{C}}$.

There is the following "smooth" version of the Kostant-Hochschild-Rosenberg theorem, [Ko], Thm. 4.6.1.1.

Proposition 2.2. The natural embedding

$$
\wedge^{\bullet} \mathcal{T} \rightarrow \mathcal{D}^{\bullet}
$$

is a quasiisomorphism of complexes. Moreover, if $\varphi \in \mathcal{D}^{n}$ is a Hochschild cocycle, then its alternation $\operatorname{Alt}(\varphi)$ is a polyvector field of $\wedge \bullet \mathcal{T}$ cohomological to $\varphi$.

Proof. Arguments of this proof will be used also in proving the next proposition. The proposition is local on $M$, so it is enough to prove it replacing $M$ by an open set $U \subset M$ having complex coordinates $x_{i}, i=1, \ldots, \operatorname{dim} M$. Any differential operator on $U$ may be uniquely presented as a polynomial in $\partial / \partial x_{i}$ with coefficients being smooth functions on $U$. Hence, $\mathcal{D}^{\bullet}$ coincides over $U$ with the complex $\mathcal{C}^{\bullet}(\mathcal{T})$. Here, for any vector bundle $E$, we denote by $\mathcal{C}^{\bullet}(E)$ the complex $(\otimes \bullet \operatorname{Sym}(E), d)$ with differential of the form

$$
\begin{gathered}
d: \otimes^{n} \operatorname{Sym}(E) \rightarrow \otimes^{n+1} \operatorname{Sym}(E), \\
d\left(a_{1} \otimes \ldots \otimes a_{n}\right)=1 \otimes a_{1} \otimes \ldots \otimes a_{n}+ \\
+\sum_{i=1}^{n}(-1)^{i} a_{1} \otimes \ldots \otimes \Delta a_{i} \otimes \ldots \otimes a_{n}+(-1)^{n+1} a_{1} \otimes \ldots \otimes a_{n},
\end{gathered}
$$

where $\Delta$ is the comultiplication in the symmetric algebra $\operatorname{Sym}(E)$ generated by the rule $\Delta(a)=a \otimes 1+1 \otimes a$ for $a \in E$.

One has the following well known statement (see, for example, the proof of Thm. 4.6.1.1. in [K[ $)$. 
Lemma 2.3. Let $E$ be a line bundle over $M$. Then the conclusion of Proposition 2.9 holds for the map

$$
\wedge^{\bullet} E \rightarrow \mathcal{C}^{\bullet}(E)
$$

Applying this lemma to $E=\mathcal{T}$ we prove the proposition.

Let $(M, \mathcal{P})$ be a smooth manifold with integrable distribution. We call an $n$-chain $\nu \in \mathcal{D}^{n}$ polarized if $\nu\left(a_{1}, \ldots, a_{n}\right)=0$ whenever $a_{1}, \ldots, a_{n} \in \mathcal{O}_{\mathcal{P}}$. We call $\nu$ strongly polarized, if $\nu\left(a_{1}, \ldots, a_{n}\right)=0$ whenever $a_{1}, \ldots, a_{n-1} \in \mathcal{O}_{\mathcal{P}}$ and $a_{n} \in \mathcal{C}_{M}^{\infty}$.

Proposition 2.4. Let $\nu \in \mathcal{D}^{2}$ be a polarized Hochschild 2-cochain such that $d \nu$ is strongly polarized. Then, there exists a polarized differential operator $b$ such that $\nu+d b$ is strongly polarized.

Proof. Since subsheaves of polarized and strongly polarized cochains are subsheaves of $\mathcal{C}_{M}^{\infty}$-modules, it is enough to prove the proposition locally on $M$. So, let $U$ be an open set with coordinates $x_{i}, i=1, \ldots, \operatorname{dim} M$ such that $\partial / \partial x_{i}, i=1, \ldots, k$, form a local frame in $\mathcal{P}$. Let $\mathcal{Q}$ be the subbundle in $\mathcal{T}$ generated by $\partial / \partial x_{i}, i=k+1, \ldots, n$. Thus, $\mathcal{T}=\mathcal{Q} \oplus \mathcal{P}$ over $U$. There is the natural isomorphism of complex $\mathcal{C}^{\bullet}(\mathcal{T})$ with the tensor product of complexes $\mathcal{C}^{\bullet}(\mathcal{Q}, \mathcal{P})=\mathcal{C}^{\bullet}(\mathcal{Q}) \otimes \mathcal{C}^{\bullet}(\mathcal{P})$, so we can identify $\mathcal{C}^{\bullet}(\mathcal{T})$ with $\mathcal{C}^{\bullet}(\mathcal{Q}, \mathcal{P})$. Similarly, we identify the complex $\wedge^{\bullet}(\mathcal{T})$ with $\wedge^{\bullet}(\mathcal{Q}, \mathcal{P})=\wedge^{\bullet}(\mathcal{Q}) \otimes \wedge^{\bullet}(\mathcal{P})$.

Thus, the map (2.2) generates the map of complexes

$$
\wedge^{\bullet}(\mathcal{Q}, \mathcal{P}) \rightarrow \mathcal{C}^{\bullet}(\mathcal{Q}, \mathcal{P})
$$

Complex $\mathcal{C}_{\bullet}(\mathcal{Q}, \mathcal{P})$ decomposes obviously into a direct sum of subcomplexes $\mathcal{C}_{k, l}(\mathcal{Q}, \mathcal{P})$, where $\mathcal{C}_{k, l}(\mathcal{Q}, \mathcal{P})$ consists of elements of total degree $k$ with respect to $\mathcal{P}$ and $l$ with respect to $\mathcal{Q}$. The same is true for $\Lambda^{\bullet}(\mathcal{Q}, \mathcal{P})$. Due to Proposition 2.2, the map (2.3) is a quasiisomorphism of bigraded complexes.

It is clear that an element of $\mathcal{C}^{n}(\mathcal{Q}, \mathcal{P})$ is polarized, if it is a sum of tensor monomials having degree $>0$ with respect to $\mathcal{P}$. An element of $\mathcal{C}^{n}(\mathcal{Q}, \mathcal{P})$ is strongly polarized if it is a sum of tensor monomials of the form $a_{1} \otimes \cdots \otimes a_{n}$ where $a_{1} \otimes \cdots \otimes a_{n-1}$ is polarized.

So, we may suppose that given $\nu$ is a polarized Hochschild cochain on $U$ belonging to $\mathcal{C}^{2}(\mathcal{Q}, \mathcal{P})$. It can be written as $\nu=\nu_{0}+\nu_{1}$, where $\nu_{0}=\sum\left(a_{i} \otimes c_{i}\right)$, the sum of all tensor monomials in $\nu$ such that $a_{i} \in \mathcal{C}^{1}(\mathcal{Q}), c_{i} \in \mathcal{C}^{1}(\mathcal{P})$. Let us denote $b=\sum a_{i} c_{i}$. It is clear that $\nu^{\prime}=\nu+d b$ does not contain tensor monomials of that type. The proposition will be proved if we show that $\nu^{\prime}$ is strongly polarized. Let us prove this. 
Suppose $\nu^{\prime}=\nu_{0}^{\prime}+\nu_{1}^{\prime}$, where $\nu_{0}^{\prime}$ is not strongly polarized and $\nu_{1}^{\prime}$ is strongly polarized. Then $\nu_{0}^{\prime}$ has the form

$$
\nu_{0}^{\prime}=\sum\left(a_{i} \otimes b_{i}\right)\left(1 \otimes c_{i}\right)
$$

where $\left(a_{i} \otimes b_{i}\right) \in \otimes^{2} \operatorname{Sym}(\mathcal{Q})$ and $c_{i} \in \operatorname{Sym}(\mathcal{P})$ are linearly independent. Besides, $b_{i}$ are of degree $>0$.

The element $d \nu=d \nu^{\prime}=d \nu_{0}^{\prime}+d \nu_{1}^{\prime}$ is strongly polarized by hypothesis of the proposition, $d \nu_{1}^{\prime}$ being the coboundary of a strongly polarized element $\nu_{1}^{\prime}$ is strongly polarized too. All summands of $d \nu_{0}^{\prime}$ with first two factors being of degree zero with respect to $\mathcal{P}$ are $\left(d\left(a_{i} \otimes b_{i}\right)+a_{i} \otimes b_{i} \otimes 1\right)\left(1 \otimes 1 \otimes c_{i}\right)$. These summands are not strongly polarized. Hence, $\sum_{i}\left(d\left(a_{i} \otimes b_{i}\right)+a_{i} \otimes b_{i} \otimes 1\right)\left(1 \otimes 1 \otimes c_{i}\right)=0$. Since elements $1 \otimes 1 \otimes c_{i}$ are linearly independent, it follows that $d\left(a_{i} \otimes b_{i}\right)=-a_{i} \otimes b_{i} \otimes 1$ for all $i$, which is only possible if $a_{i} \otimes b_{i}=0$ for all $i$. It follows from (2.4) that $\nu_{0}^{\prime}=0$. So, $\nu^{\prime}$ is equal to $\nu_{1}^{\prime}$ which is strongly polarized.

\subsection{Differential operators in presence of a distribution}

Let $(M, \mathcal{P})$ be a smooth manifold with integrable distribution. Let $z_{i}, y_{j}$ be complex coordinates on an open set $U \subset M$ such that $\mathcal{P}=\left(d z_{i}\right)^{\perp}$. Vector fields $\partial / \partial y_{j}$ form a local frame in $\mathcal{P}$, since, by definition, $\mathcal{O}_{\mathcal{P}}$ consists of functions $a \in \mathcal{C}_{M}^{\infty}$ such that $d a$ has the form $\sum_{i} a_{i} d z_{i}$. Since $d a$ is closed, $\partial a_{i} / \partial y_{j}=0$ for all $i, j$, which implies that $a_{i}=\partial a / \partial z_{i} \in \mathcal{O}_{\mathcal{P}}$ for all $i$.

Let $\mathcal{Q}$ be a subbundle in $\mathcal{T}=\mathcal{T}_{M}^{\mathbb{C}}$ generated by $\partial / \partial z_{j}$, so that $\mathcal{T}=\mathcal{Q} \oplus \mathcal{P}$ over $U$.

The vector bundle $\mathcal{T} / \mathcal{P}$ may be considered as the sheaf of derivations from $\mathcal{O}_{\mathcal{P}}$ to $\mathcal{C}_{M}^{\infty}$, $\operatorname{Der}\left(\mathcal{O}_{\mathcal{P}}, \mathcal{C}_{M}^{\infty}\right)$. Locally, such derivations can be presented in the form $\sum b_{i} \partial / \partial z_{i}, b_{i} \in \mathcal{C}_{M}^{\infty}$, i.e. as sections of $\mathcal{Q}$. Denote by $\operatorname{Der}\left(\mathcal{O}_{\mathcal{P}}\right)$ the $\mathcal{O}_{\mathcal{P}}$-submodule of $\operatorname{Der}\left(\mathcal{O}_{\mathcal{P}}, \mathcal{C}_{M}^{\infty}\right)$ consisting of operators which take $\mathcal{O}_{\mathcal{P}}$ to itself. It is clear that $\operatorname{Der}\left(\mathcal{O}_{\mathcal{P}}, \mathcal{C}_{M}^{\infty}\right)=\mathcal{C}_{M}^{\infty} \otimes_{\mathcal{O}_{\mathcal{P}}} \operatorname{Der}\left(\mathcal{O}_{\mathcal{P}}\right)$. Locally, elements of $\operatorname{Der}\left(\mathcal{O}_{\mathcal{P}}\right)$ have the form $\sum_{i} a_{i} \partial / \partial z_{i}, a_{i} \in \mathcal{O}_{\mathcal{P}}$.

Let $\wedge^{\bullet}(\mathcal{T} / \mathcal{P})$ denote the complex of sheaves of polyvector fields on $M$ from $\mathcal{O}_{\mathcal{P}}$ to $\mathcal{C}_{M}^{\infty}$.

Let $\mathcal{D}^{\bullet}\left(\mathcal{O}_{\mathcal{P}}, \mathcal{C}_{M}^{\infty}\right)$ denote the restriction of the Hochschild complex $\mathcal{D}^{\bullet}$ to $\mathcal{O}_{\mathcal{P}}$. So, the sheaf $\mathcal{D}^{n}\left(\mathcal{O}_{\mathcal{P}}, \mathcal{C}_{M}^{\infty}\right)$ may be considered as the sheaf of $n$-differential operators from $\mathcal{O}_{\mathcal{P}}$ to $\mathcal{C}_{M}^{\infty}$. Locally, elements of $\mathcal{D}^{1}\left(\mathcal{O}_{\mathcal{P}}, \mathcal{C}_{M}^{\infty}\right)$, the sheaf of differential operators from $\mathcal{O}_{\mathcal{P}}$ to $\mathcal{C}_{M}^{\infty}$, can be presented as polynomials in $\partial / \partial z_{i}$ with smooth coefficients. So, locally on $M$, complex $\mathcal{D}^{\bullet}\left(\mathcal{O}_{\mathcal{P}}, \mathcal{C}_{M}^{\infty}\right)$ is isomorphic to the complex $\mathcal{C}^{\bullet}(\mathcal{Q})$ (see previous subsection).

We will need the following version of the Kostant-Hochschild-Rosenberg theorem.

Proposition 2.5. The natural embedding

$$
\wedge^{\bullet}(\mathcal{T} / \mathcal{P}) \rightarrow \mathcal{D}^{\bullet}\left(\mathcal{O}_{\mathcal{P}}, \mathcal{C}_{M}^{\infty}\right)
$$


is a quasiisomorphism of complexes. Moreover, if $\varphi \in \mathcal{D}^{n}\left(\mathcal{O}_{\mathcal{P}}, \mathcal{C}_{M}^{\infty}\right)$ is a cocycle, then Alt $(\varphi)$ is a polyvector field of $\wedge^{n}(\mathcal{T} / \mathcal{P})$ cohomological to $\varphi$.

Proof. As follows from above, embedding (2.5) is locally isomorphic to the embedding

$$
\wedge^{\bullet} \mathcal{Q} \rightarrow \mathcal{C}^{\bullet}(\mathcal{Q})
$$

Now the proposition follows from Lemma 2.3 when $E=\mathcal{Q}$.

Remark 2.6. All conclusions of Propositions 2.2, 2.4, 2.5 remain true for global sections of the corresponding sheaves, since they are sheaves of $\mathcal{C}_{M}^{\infty}$-modules.

\subsection{Differential forms in presence of a distribution}

Let $(M, \mathcal{P})$ be a smooth manifold with integrable distribution.

The sheaf $\mathcal{P}^{\perp}=(\mathcal{T} / \mathcal{P})^{*}$ of differential forms on $M$ which being applied to vector fields from $\mathcal{P}$ give zero, may be written as $\mathcal{C}_{M}^{\infty} d \mathcal{O}_{\mathcal{P}}$.

Denote $\Omega_{\mathcal{O}_{\mathcal{P}}}^{1}=\operatorname{Hom}_{\mathcal{O}_{\mathcal{P}}}\left(\operatorname{Der}\left(\mathcal{O}_{\mathcal{P}}\right), \mathcal{O}_{\mathcal{P}}\right)$, the sheaf of 1 -forms on $\mathcal{O}_{\mathcal{P}}$. It is clear that $\Omega_{\mathcal{O}_{\mathcal{P}}}^{1}=\mathcal{O}_{\mathcal{P}} d \mathcal{O}_{\mathcal{P}}$

Denote by $\Omega_{\mathcal{O}_{\mathcal{P}}}^{1 c l}$ the subsheaf of closed forms of $\Omega_{\mathcal{O}_{\mathcal{P}}}^{1}$.

Lemma 2.7. a) The sequence of sheaves

$$
0 \longrightarrow \mathbb{C} \longrightarrow \mathcal{O}_{\mathcal{P}} \stackrel{d}{\longrightarrow} \Omega_{\mathcal{O}_{\mathcal{P}}}^{1 c l} \longrightarrow 0
$$

is exact.

b) The sequence of sheaves

$$
0 \longrightarrow \Omega_{\mathcal{O}_{\mathcal{P}}}^{1 c l} \longrightarrow \mathcal{P}^{\perp} \stackrel{d}{\longrightarrow} d \mathcal{P}^{\perp} \longrightarrow 0
$$

is exact.

Proof. Let functions $z_{i}, y_{j}$ form a local basis on $M$ and $\mathcal{P}=\left(d z_{i}\right)^{\perp}$. Let us prove a). It is sufficient to establish the exactness at the third term of (2.6). Let $\alpha=\sum_{i} a_{i} d z_{i} \in \Omega_{\mathcal{O}_{\mathcal{P}}}^{1 c l}$. Since $\alpha$ is closed, there exists, locally, $f \in \mathcal{C}_{M}^{\infty}$ such that $d f=\alpha$. Since $\alpha$ does not contain terms of the form $g d y_{j}$, one has $\partial f / \partial y_{j}=0$ for all $j$. Hence, $f \in \mathcal{O}_{\mathcal{P}}$.

To prove b), it is sufficient to establish the exactness at $\mathcal{P}^{\perp}$. To this end, suppose $\beta=\sum_{i} b_{i} d z_{i} \in \mathcal{P}^{\perp}$ and $d \beta=0$. Since $\beta$ is closed, $\partial b_{i} / \partial y_{j}=0$ for all $i, j$. This means that all $b_{i} \in \mathcal{O}_{\mathcal{P}}$. Thus, $\beta \in \Omega_{\mathcal{O}_{\mathcal{P}}}^{1}$ and closed, i.e. $\beta \in \Omega_{\mathcal{O}_{\mathcal{P}}}^{1 c l}$. 
Proposition 2.8. Let $(M, \mathcal{P})$ be a smooth manifold with an integrable distribution. Then, there is the natural isomorphism

$$
H^{1}\left(M, \Omega_{\mathcal{O}_{\mathcal{P}}}^{1 c l}\right) \simeq \Gamma\left(M, d \mathcal{P}^{\perp}\right) / d\left(\Gamma\left(M, \mathcal{P}^{\perp}\right)\right)
$$

Proof. This is an immediate consequence of the cohomological exact sequence for (2.7) and of $H^{i}\left(M, \mathcal{P}^{\perp}\right)=0$ for $i>0$.

\section{$3 \quad \mathbb{C}$-symplectic manifolds and their polarizations}

\section{$3.1 \quad \mathbb{C}$-symplectic manifolds}

Definition 3.1. By a $\mathbb{C}$-symplectic manifold we mean a pair $(M, \omega)$, where $M$ is a smooth manifold and $\omega$ a closed nondegenerate complex 2-form on $M$ satisfying the following condition: each point of $M$ has a neighborhood $U$ and complex coordinates $x_{i}, y_{i}, i=$ $1, \ldots, \frac{1}{2} \operatorname{dim} M$, on $U$ such that the form $\omega$ on $U$ can be presented as

$$
\omega=\sum_{i} d y_{i} \wedge d x_{i}
$$

If the form $\omega$ is real, such a presentation is possible by the Darboux theorem. One has

$$
\left\{x_{i}, x_{j}\right\}=\left\{y_{i}, y_{j}\right\}=0, \quad\left\{y_{i}, x_{j}\right\}=\delta_{i j}
$$

for all $i, j$, where $\{\cdot, \cdot\}$ is the Poisson bracket inverse to $\omega$. We call such functions $x_{i}, y_{i}$ Darboux coordinates with respect to $\omega$ (or $\{\cdot, \cdot\}$ ).

In what follows we only deal with $\mathbb{C}$-symplectic manifolds, so we simply call them symplectic ones.

\subsection{Polarization}

Definition 3.2. Let $(M, \omega)$ be a symplectic manifold. We call a (complex) distribution $\mathcal{P}$ on $M$ a polarization of $\omega$, if, locally, there exist (complex) Darboux coordinates, $x_{i}, y_{i}$, with respect to $\omega$ such that $\mathcal{P}=\left(d x_{i}\right)^{\perp}$, i.e. $x_{i} \in \mathcal{O}_{\mathcal{P}}$.

We call the triple $(M, \omega, \mathcal{P})$ a polarized symplectic manifold $(\mathrm{PSM})$.

It follows that a polarization of $\omega$ is, in particular, an integrable distribution and a Lagrangian subbundle with respect to $\omega$.

Proposition 3.3. Let $(M, \omega, \mathcal{P})$ be a PSM. Then $\omega \in \Gamma\left(M, d \mathcal{P}^{\perp}\right)$. 
Proof. Let $x_{i}, y_{i}$ be local Darboux coordinates on $M$ such that $\mathcal{P}=\left(d x_{i}\right)^{\perp}$ and $\omega=$ $\sum d y_{i} \wedge d x_{i}$. Then, locally, $\omega=d\left(\sum y_{i} d x_{i}\right)$ and $\sum y_{i} d x_{i} \in \mathcal{P}^{\perp}$.

Proposition 3.4. Let $(M, \omega, \mathcal{P})$ be a PSM. Then, $\mathcal{O}_{\mathcal{P}}$ is a maximal commutative Lie subalgebra in $\mathcal{C}_{M}^{\infty}$ with respect to the Poisson bracket $\omega^{-1}$.

Proof. It follows from Definition 3.2 that, locally, the bracket $\omega^{-1}$ may be written in the form

$$
\{\cdot, \cdot\}=\sum_{i} \bar{\partial}_{i} \wedge \partial_{i},
$$

where $\partial_{i}=\left\{y_{i}, \cdot\right\}, \bar{\partial}_{i}=\left\{\cdot, x_{i}\right\}$. The module $\mathcal{O}_{\mathcal{P}}$ consists, locally, of elements $g \in \mathcal{C}_{M}^{\infty}$ such that $\left\{g, x_{i}\right\}=\bar{\partial}_{i} g=0$ for all $i$. Putting two such elements $g_{1}, g_{2}$ in (3.3), we obtain that $\left\{g_{1}, g_{2}\right\}=0$. So $\mathcal{O}_{\mathcal{P}}$ is commutative. The maximality of $\mathcal{O}_{\mathcal{P}}$ is obvious. Indeed, if $a \in \mathcal{C}_{M}^{\infty}$ commutes with $\mathcal{O}_{\mathcal{P}}$, then, in particular, $\left\{x_{i}, a\right\}=0$ for all $i$, hence $a \in \mathcal{O}_{\mathcal{P}}$.

\section{Deformations of a polarized symplectic manifold}

\subsection{Formal everything}

Let $t$ be a formal parameter. We will consider on $M$ formal functions, formal vector fields, formal forms, etc., which are elements of $\mathcal{C}_{M}^{\infty}[[t]], \mathcal{T}_{M}^{\mathbb{C}}[[t]], \Phi^{k}[[t]]$, etc. In the formal case all sheaves over $M$ and their morphisms will be sheaves and morphisms of $\mathbb{C}[[t]]$-modules.

Let $\mathcal{B}$ be a sheaf over $M$. We call the map $\sigma: \mathcal{B}[[t]] \rightarrow \mathcal{B}, b_{0}+t b_{1}+\cdots \rightarrow b_{0}$, the symbol map. For a subsheaf $\mathcal{F}_{t} \subset \mathcal{B}[[t]]$, we denote $\mathcal{F}_{0}=\sigma\left(\mathcal{F}_{t}\right) \subset \mathcal{B}$.

Let $\mathcal{F}_{t}$ be a subsheaf of $\mathcal{B}[[t]]$. We call $\mathcal{F}_{t}$ a $t$-regular subsheaf if it is complete in $t$-adic topology, and $t b \in \mathcal{F}_{t}, b \in \mathcal{B}[[t]]$ implies $b \in \mathcal{F}_{t}$.

For a $t$-regular subsheaf $\mathcal{F}_{t} \subset \mathcal{B}[[t]]$ the natural map $\mathcal{F}_{t} / t \mathcal{F}_{t} \rightarrow \mathcal{F}_{0}$ is an isomorphism. In this case we call $\mathcal{F}_{t}$ a deformation of $\mathcal{F}_{0}$.

Let $\mathcal{B}$ be a vector bundle over $M$. Then, a subsheaf $\mathcal{F}_{t} \subset \mathcal{B}[[t]]$ is called a (formal) subbundle, if it is a $t$-regular subsheaf of $\mathcal{C}_{M}^{\infty}[[t]]$-modules.

All notions and statements above carry over to the formal case.

For example, a deformation of a distribution $\mathcal{P}$ on $M$ is a subbundle, $\mathcal{P}_{t}$, of $\mathcal{T}_{M}^{\mathbb{C}}[[t]]$ such that $\mathcal{P}_{0}=\mathcal{P}$. A system of (formal) coordinates on an open set $U \subset M$ is a set of formal functions $x_{i}=x_{i 0}+t x_{1 i}+\cdots, i=1, \ldots, \operatorname{dim} M, \mathbb{C}[[t]]$-linearly independent at each point of $U$. A formal polarized symplectic manifold is a triple $\left(M, \omega_{t}, \mathcal{P}_{t}\right)$, where $\omega_{t}$ is a 
formal symplectic form on $M$ (i.e. a closed 2-form of the form $\omega_{t}=\omega_{0}+t \omega_{1}+\cdots$ with nondegenerate $\omega_{0}$ ) and $\mathcal{P}_{t}$ is a polarization of $\omega_{t}$ in sense of Definition 3.2, i.e., locally, there exist formal Darboux coordinates $x_{i}, y_{i}$ with respect to $\omega_{t}$ such that $\mathcal{P}_{t}=\left(d x_{i}\right)^{\perp}$.

We say that $\left(M, \omega_{t}, \mathcal{P}_{t}\right)$ is a deformation of a polarized symplectic manifold $(M, \omega, \mathcal{P})$, if $\left(M, \omega_{0}, \mathcal{P}_{0}\right)=(M, \omega, \mathcal{P})$.

The following proposition, which follows from Propositions 6.1 and 6.2 below, shows that any deformation of (polarized) symplectic manifold is a formal (polarized) symplectic manifold.

Proposition 4.1. a) Let $\omega_{t}$ be a formal closed 2-form. If $\omega_{0}$ admits, locally, Darboux coordinates, then there exist their lifts being formal Darboux coordinates for $\omega_{t}$.

b) Let $\mathcal{P}_{t}$ be an integrable distribution and Lagrangian with respect to $\omega_{t}$. Let $\mathcal{P}_{0}$ be a polarization of $\omega_{0}$. Then $\mathcal{P}_{t}$ is a polarization of $\omega_{t}$.

\subsection{Local structure of deformed polarizations}

It is clear that formal vector fields $t X_{1}+t^{2} X_{2}+\cdots \in t \mathcal{T}_{M}^{\mathbb{C}}[[t]]$ form a sheaf of pro-nilpotent Lie algebras. It follows that elements $e^{t X}, X \in \mathcal{T}_{M}^{\mathbb{C}}[[t]]$, form a sheaf of pro-unipotent Lie groups of formal automorphisms of $M$.

Let $x_{i}$ be formal coordinates on $U$ and $a_{i}, i=1, \ldots, \operatorname{dim} M$, arbitrary formal functions on $U$. Then there exists a derivation, $D$, of $\mathcal{C}_{M}^{\infty}[[t]]$ that takes $x_{i}$ to $a_{i}$. Such a derivation is $D=\sum_{i} a_{i} \partial / \partial x_{i}$. This implies the following

Lemma 4.2. Let $x_{i}, x_{i}^{\prime}, i=1, \ldots, \operatorname{dim} M$, be two systems of formal coordinates on an open set $U \subset M$, and $x_{i}=x_{i}^{\prime} \bmod t$. Then, there exists a formal automorphism on $U$ that takes $x_{i}$ to $x_{i}^{\prime}$.

Proposition 4.3. a) Let $\mathcal{P}_{t}$ be an integrable distribution on $M$ which is a deformation of a distribution $\mathcal{P}$. Then, locally, there exists a formal vector field, $X$, such that $e^{t X}$ gives an isomorphism $\mathcal{P}_{t}$ with $\mathcal{P}[[t]]$.

b) Let $\left(M, \omega_{t}, \mathcal{P}_{t}\right)$ be a deformation of a polarized symplectic manifold $(M, \omega, \mathcal{P})$. Then, each point of $M$ has a neighborhood, $U$, and a formal vector field $X$ on $U$ such that $e^{t X}$ gives an isomorphism of $\left.\left(M, \omega_{t}, \mathcal{P}_{t}\right)\right|_{U}$ with the trivial deformation $(U, \omega, \mathcal{P}[[t]])$.

Proof. a) Locally, there exist functions $x_{i t}=x_{i 0}+t x_{i 1}+\cdots, i=1, \ldots, k$, such that $\mathcal{P}_{t}=\left(d x_{i t}\right)^{\perp}$ and hence $\mathcal{P}=\left(d x_{i 0}\right)^{\perp}$. Let us add functions $x_{(k+1) 0}, \ldots, x_{n 0}$ in such a way that all $x_{i 0}, i=1, \ldots, n$, form a coordinate system. According to Lemma 4.2 , there exists 
a formal automorphism which takes coordinates $x_{i t}, i=1, \ldots, k, x_{j 0}, j=k+1, \ldots, n$, to coordinates $x_{i 0}, i=1, \ldots, n$. This formal automorphism gives obviously an isomorphism $\mathcal{P}_{t}$ onto $\mathcal{P}[[t]]$.

b) Let $U$ be an open set in $M$ where Darboux coordinates $x_{i t}=x_{i 0}+t x_{i 1}+\cdots$, $y_{i t}=y_{i 0}+t x_{i 1}+\cdots, i=1, \ldots, \frac{1}{2} \operatorname{dim} M$, exist, and $\mathcal{P}_{t}=\left(d x_{i t}\right)^{\perp}$. Then, $x_{i 0}, y_{i 0}$ are Darboux coordinates with respect to $\omega_{0}=\omega$ such that $\mathcal{P}=\left(d x_{i 0}\right)^{\perp}$. By Lemma 4.2 there exists a formal vector field $X$ on $U$ such that the formal automorphism $e^{t X}$ takes coordinates $x_{i t}, y_{i t}$ to $x_{i 0}, y_{i 0}$. Such $X$ satisfies the conclusion of the proposition.

Let $\mathcal{P}$ be an integrable distribution. Denote by $\mathcal{O}_{\mathcal{P}}$ the sheaf of functions constant along $\mathcal{P}$. Let $\mathcal{P}_{t}$ be a deformation of $\mathcal{P}$. It follows from the previous proposition that, locally, the pair $\left(\mathcal{C}_{M}^{\infty}[[t]], \mathcal{O}_{\mathcal{P}_{t}}\right)$ is isomorphic to the pair $\left(\mathcal{C}_{M}^{\infty}[[t]], \mathcal{O}_{\mathcal{P}}[[t]]\right)$, hence $\mathcal{O}_{\mathcal{P}_{t}}$ is a $t$-regular subalgebra of $\mathcal{C}_{M}^{\infty}[[t]]$. One holds the following inverse assertion.

Proposition 4.4. Let $\mathcal{P}$ be an integrable distribution on $M$. Let $\mathcal{O}_{t}$ be a t-regular subalgebra of $\mathcal{C}_{M}^{\infty}[[t]]$ such that $\mathcal{O}_{0}=\mathcal{O}_{\mathcal{P}}$. Then, there exists a deformation, $\mathcal{P}_{t}$, of $\mathcal{P}$ such that $\mathcal{O}_{t}=\mathcal{O}_{\mathcal{P}_{t}}$

Proof. Let us prove that $d \mathcal{O}_{t} \subset\left(\mathcal{T}_{M}^{\mathbb{C}}\right)^{*}[[t]]$ is $t$-regular. Let $b \in\left(\mathcal{T}_{M}^{\mathbb{C}}\right)^{*}[[t]]$ and $t b \in d \mathcal{O}_{t}$. Then, there exists $a=a_{0}+t a^{\prime} \in \mathcal{O}_{t}$ such that $d a=t b$. It follows that $a_{0}$ is a constant, so $t a^{\prime} \in \mathcal{O}_{t}$. Since $\mathcal{O}_{t}$ is $t$-regular, $a^{\prime} \in \mathcal{O}_{t}$, too. Therefore, $b=d a^{\prime} \in d \mathcal{O}_{t}$, so that $d \mathcal{O}_{t}$ is a $t$ regular submodule in $\left(\mathcal{T}_{M}^{\mathbb{C}}\right)^{*}[[t]]$. This implies that $\mathcal{C}_{M}^{\infty}[[t]] d \mathcal{O}_{t}$ is a subbundle in $\left(\mathcal{T}_{M}^{\mathbb{C}}\right)^{*}[[t]]$, so $\mathcal{P}_{t}=\left(d \mathcal{O}_{t}\right)^{\perp}$ is a subbundle in $\left.\mathcal{T}_{M}^{\mathbb{C}}[t]\right]$. Moreover, $\mathcal{P}_{0}=\mathcal{P}$. So, $\mathcal{P}_{t}$ is a deformation of $\mathcal{P}$. One has $\mathcal{O}_{t} \subset \mathcal{O}_{\mathcal{P}_{t}}$. Since these two subalgebras are $t$-regular and coincide at $t=0$, we have $\mathcal{O}_{t}=\mathcal{O}_{\mathcal{P}_{t}}$.

The last proposition shows that there is a one-to-one correspondence between deformations of an integrable distribution $\mathcal{P}$ and deformations of $\mathcal{O}_{\mathcal{P}}$.

\subsection{Action of formal automorphisms on a symplectic form}

Let $\left(M, \omega_{t}\right)$ be a formal symplectic manifold, $\mathcal{T}=\mathcal{T}_{M}^{\mathbb{C}}$.

The well known formula for the Lie derivative

$$
L_{X}=i(X) d+\operatorname{di}(X)
$$

implies

$$
L_{X} \omega_{t}=d \alpha(X)
$$


where $\alpha: \mathcal{T}[[t]] \rightarrow \mathcal{T}^{*}[[t]]$ is the map defined by $X \mapsto \omega_{t}(X, \cdot)$. Since $\alpha$ is an isomorphism, we have the following lemma.

Lemma 4.5. The orbit of $\omega_{t}$ by the action of the group of formal automorphisms $e^{t X}$, $X \in \Gamma(M, \mathcal{T}[[t]])$, is $\omega_{t}+t d\left(\Gamma\left(M, \mathcal{T}^{*}[[t]]\right)\right)$.

The lemma shows that the orbit of $\omega_{t}$ coincides with the cohomology class of $\omega_{t}$ in $\omega_{0}+t H^{2}(M, \mathbb{C}[[t]])$.

Let $\left(M, \omega_{t}, \mathcal{P}_{t}\right)$ be a formal PSM and $X \in \Gamma\left(M, \mathcal{P}_{t}\right)$. Since $\alpha\left(\mathcal{P}_{t}\right)=\mathcal{P}_{t}^{\perp}$, one has $L_{X} \omega_{t}=d \alpha(X) \in \Gamma\left(M, d \mathcal{P}_{t}^{\perp}\right)$. The argument as above implies

Lemma 4.6. By the action of the group generated by $e^{t X}, X \in \Gamma\left(M, \mathcal{P}_{t}\right)$, the orbit of $\omega_{t}$ is $\omega_{t}+t d\left(\Gamma\left(M, \mathcal{P}_{t}^{\perp}\right)\right)$.

The lemma shows that the orbit of $\omega_{t}$ corresponds to the cohomology class of $\omega_{t}$ in $\Gamma\left(M, d \mathcal{P}_{t}^{\perp}\right) / d\left(\Gamma\left(M, \mathcal{P}_{t}^{\perp}\right)\right)$.

\section{$5 \quad$ Polarized symplectic connection and characteristic class of a polarized symplectic manifold}

\subsection{Polarized symplectic connection}

Let $(M, \omega, \mathcal{P})$ be a (formal) PSM. Denote, for shortness, $\mathcal{T}=\mathcal{T}_{M}^{\mathbb{C}}[[t]]$.

Definition 5.1. We call a connection, $\nabla$, on $M$ a $\mathcal{P}$-symplectic connection if

a) it preserves $\omega$ and is torsion free, i.e. is a symplectic connection;

b) it preserves $\mathcal{P}$, i.e. $\nabla_{X}(\mathcal{P}) \subset \mathcal{P}$ for any $X \in \mathcal{T}$;

c) it is flat on $\mathcal{P}$ along $\mathcal{P}$, i.e. for any $X, Y \in \mathcal{P}$ one has $\left(\nabla_{X} \nabla_{Y}-\nabla_{Y} \nabla_{X}-\nabla_{[X, Y]}\right)(\mathcal{P})=$ 0 .

Proposition 5.2. Let $(M, \omega, \mathcal{P})$ be a (formal) PSM. Then, there exists a $\mathcal{P}$-symplectic connection on $M$.

Proof. Let functions $a_{1}, \ldots, a_{2 n}, 2 n=\operatorname{dim} M$, form local Darboux coordinates on an open set $U \subset M$ and be such that $a_{i} \in \mathcal{O}_{\mathcal{P}}$ for $i=1, \ldots, n$. Let $X_{i}=X_{a_{i}}$ be the corresponding Hamiltonian vector fields. Then, vector fields $X_{i}, i=1, \ldots, n$, form a local frame in $\mathcal{P}$. Also, all $X_{i}$ commute and form a local frame in $\mathcal{T}$. Let $\nabla$ be the standard flat connection on $U$ associated with coordinates $a_{i}$. This connection is defined on $U$ by the rule $\nabla_{X_{i}} X_{j}=0$. 
It is easy to see that $\nabla$ is a $\mathcal{P}$-symplectic connection on $U$. Moreover, since $X_{f} \in \mathcal{P}$ is equivalent to $d f \in \mathcal{P}^{\perp}$, the connection $\nabla$ satisfies the following property for Hamiltonian vector fields:

$$
\nabla_{X_{f}} X_{g}=0 \quad \text { for } X_{f}, Y_{g} \in \mathcal{P} .
$$

Now, let us prove the existence of a global connection.

Let $\left\{U_{\alpha}\right\}$ is an open covering of $M$ such that on each $U_{\alpha}$ there is a $\mathcal{P}$-symplectic connection $\nabla_{\alpha}$ as above. Then $\nabla_{\alpha}-\nabla_{\beta}$ defined on $U_{\alpha} \cap U_{\beta}$ form a Čech cocycle $\psi_{\alpha, \beta} \in$ $\operatorname{Hom}(\mathcal{T} \otimes \mathcal{T}, \mathcal{T}), \psi_{\alpha, \beta}(X, Y)=\nabla_{\alpha X} Y-\nabla_{\beta X} Y$

Elements $\psi_{\alpha, \beta}$ satisfy the following properties.

As follows from (5.1),

$$
\psi_{\alpha, \beta}(X, Y)=0 \quad \text { for } X, Y \in \mathcal{P} .
$$

Since all $\nabla_{\alpha}$ are torsion free, $\psi_{\alpha, \beta}$ are symmetric. Since all $\nabla_{\alpha}$ preserve $\mathcal{P}$, one has $\psi_{\alpha, \beta}(X, Y) \in \mathcal{P}$ for $Y \in \mathcal{P}$.

In addition, $\psi_{\alpha, \beta}$ considered as elements from $\operatorname{Hom}(\mathcal{T}, \operatorname{Hom}(\mathcal{T}, \mathcal{T})), X \mapsto \psi_{\alpha, \beta}(X, \cdot)$, belong to $\operatorname{Hom}(\mathcal{T}, \mathfrak{s p}(\mathcal{T})$ ), where $\mathfrak{s p}(\mathcal{T})$ consists of endomorphisms of $\mathcal{T}$ preserving $\omega$.

Since all the properties above are $\mathcal{C}_{M}^{\infty}[[t]]$-linear, one can find tensors $\psi_{\alpha} \in \operatorname{Hom}(\mathcal{T} \otimes$ $\mathcal{T}, \mathcal{T}$ ) satisfying all of them and such that $\psi_{\alpha}-\psi_{\beta}=\psi_{\alpha, \beta}$. Then $\nabla=\nabla_{\alpha}-\psi_{\alpha}=\nabla_{\beta}-\psi_{\beta}$ is a globally defined connection. Flatness of $\nabla$ on $\mathcal{P}$ along $\mathcal{P}$ follows from the fact that for all $\alpha$ tensors $\psi_{\alpha}$ satisfy property (5.2), i.e. $\psi_{\alpha}(X, Y)=0$ for $X, Y \in \mathcal{P}$. Also, $\nabla$ is torsion free because all $\psi_{\alpha}$ are symmetric. So, $\nabla$ satisfies the proposition.

\subsection{Characteristic class of a polarized symplectic manifold}

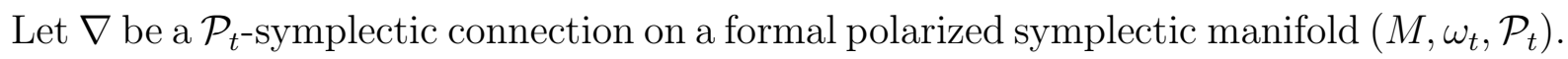
Let us denote by $\left.\nabla^{2}\right|_{\mathcal{P}_{t}}$ the curvature of $\nabla$ restricted to $\mathcal{P}_{t}$. Then, $\operatorname{tr}\left(\left.\nabla^{2}\right|_{\mathcal{P}_{t}}\right)$ is a closed 2 -form on $M$ that represents, up to a constant factor, the first Chern class of $\mathcal{P}_{0}$.

Lemma 5.3. Let $\nabla$ be a $\mathcal{P}_{t}$-symplectic connection. Then $\operatorname{tr}\left(\left.\nabla^{2}\right|_{\mathcal{P}_{t}}\right)$ belongs to $\Gamma\left(M, d \mathcal{P}_{t}^{\perp}\right)$. If $\nabla_{1}$ is another $\mathcal{P}_{t}$-symplectic connection, then $\operatorname{tr}\left(\left.\nabla_{1}^{2}\right|_{\mathcal{P}_{t}}\right)$ differs from $\operatorname{tr}\left(\left.\nabla^{2}\right|_{\mathcal{P}_{t}}\right)$ by an element of $d\left(\Gamma\left(M, \mathcal{P}_{t}^{\perp}\right)\right)$.

Proof. Follows from flatness of $\mathcal{P}_{t}$ along $\mathcal{P}_{t}$ with respect to the connection.

The lemma allows us to consider the element of $\Gamma\left(M, d \mathcal{P}_{t}^{\perp}\right) / d\left(\Gamma\left(M, \mathcal{P}_{t}^{\perp}\right)\right)$ represented by the form $\operatorname{tr}\left(\left.\nabla^{2}\right|_{\mathcal{P}_{t}}\right)$, where $\nabla$ is a $\mathcal{P}_{t^{-}}$-symplectic connection, as a characteristic class of the polarized symplectic manifold $\left(M, \omega_{t}, \mathcal{P}_{t}\right)$. 
Due to Proposition 2.8, one can also consider this class as an element of $H^{1}\left(M, \Omega_{\mathcal{O}_{\mathcal{P} t}}^{1 c l}\right)$.

\section{Deformations of Poisson brackets}

In this section we prove three technical statements which we use through the paper.

Let $\pi_{0}=\{\cdot, \cdot\}$ be a nondegenerate Poisson bracket on a smooth manifold $M$ of dimension $2 n$. We say that a formal sum $\pi_{t}=\pi_{0}+t \pi_{1}+\cdots$ is a deformation of $\pi_{0}$ if all $\pi_{i}$ are bidifferential operators on $M$ and $\pi_{t}$ defines a Lie algebra structure on the sheaf $\mathcal{C}_{M}^{\infty}[[t]]$. We will also denote $\pi_{t}$ by $[\cdot, \cdot]$. Let us recall the symbol map $\sigma: \mathcal{C}_{M}^{\infty}[[t]] \rightarrow \mathcal{C}_{M}^{\infty}$, $a=a_{0}+t a_{1}+\cdots \mapsto a_{0}$. We call $a$ a lift of $a_{0}$. We say that functions $\hat{x}_{i}, \hat{\xi}_{i}, i=1, \ldots, n$ on an open set $U \subset M$ form Darboux coordinates with respect to $[\cdot, \cdot]$, if $\left[\hat{x}_{j}, \hat{x}_{k}\right]=\left[\hat{\xi}_{j}, \hat{\xi}_{k}\right]=0$, $\left[\hat{\xi}_{j}, \hat{x}_{k}\right]=\delta_{j k}$ for all $j, k$. It is clear that then functions $x_{i}=\sigma\left(\hat{x}_{i}\right), \xi_{i}=\sigma\left(\hat{\xi}_{i}\right)$ form Darboux coordinates with respect to $\{\cdot, \cdot\}$.

Proposition 6.1. Let $[\cdot, \cdot]$ be a deformation of a Poisson bracket $\{\cdot, \cdot\}$ on $M$. Let $\hat{x}_{1}^{(i)} \ldots, \hat{x}_{n}^{(i)}, \hat{\xi}_{1}^{(i)}, \ldots, \hat{\xi}_{n}^{(i)} \in \mathcal{C}_{M}^{\infty}[[t]], i=1,2$, be two systems of Darboux coordinates with respect to $[\cdot, \cdot]$ on a contractible open set $U$ in $M$ satisfying

$$
\sigma\left(\hat{x}_{j}^{(1)}\right)=\sigma\left(\hat{x}_{j}^{(2)}\right), \quad \sigma\left(\hat{\xi}_{j}^{(1)}\right)=\sigma\left(\hat{\xi}_{j}^{(2)}\right)
$$

Then, there exists $B \in \mathcal{C}_{M}^{\infty}[[t]]$ on $U$ such that the automorphism $\Phi=\exp (t \cdot a d(B))$, where $\operatorname{ad}(B)=[B, \cdot]$, satisfies $\Phi\left(\hat{x}_{j}^{(1)}\right)=\hat{x}_{j}^{(2)}$ and $\Phi\left(\hat{\xi}_{j}^{(1)}\right)=\hat{\xi}_{j}^{(2)}$.

Proof. Let $B_{0}=0$. Assume that $B_{m}$ is such that the automorphism $\Phi_{m}=\exp \left(t \cdot a d\left(B_{m}\right)\right)$ satisfies the conclusion of the proposition modulo $t^{m+1}$. This assumption is valid for $m=0$.

Then, $\hat{x}_{j}^{(2)}=\Phi_{m}\left(\hat{x}_{j}^{(1)}\right)+t^{m+1} y_{j} \bmod t^{m+2}, \hat{\xi}_{j}^{(2)}=\Phi_{m}\left(\hat{\xi}_{j}^{(1)}\right)+t^{m+1} \eta_{j} \bmod t^{m+2}$ for suitable $y_{j}, \eta_{j} \in \mathcal{C}_{M}^{\infty}$. The Darboux commutation relations for $\hat{x}_{1}^{(i)} \ldots, \hat{x}_{n}^{(i)}, \hat{\xi}_{1}^{(i)}, \ldots, \hat{\xi}_{n}^{(i)}$ imply that the functions $y_{1}, \ldots, y_{n}, \eta_{1} \ldots, \eta_{n}$ satisfy $\left\{x_{j}, y_{k}\right\}-\left\{x_{k}, y_{j}\right\}=0,\left\{\xi_{j}, \eta_{k}\right\}-$ $\left\{\xi_{k}, \eta_{j}\right\}=0,\left\{\xi_{j}, y_{k}\right\}-\left\{x_{k}, \eta_{j}\right\}=0$, where $x_{j}=\sigma\left(\hat{x}_{j}^{(1)}\right)=\sigma\left(\hat{x}_{j}^{(2)}\right), \xi_{j}=\sigma\left(\hat{\xi}_{j}^{(1)}\right)=\sigma\left(\hat{\xi}_{j}^{(2)}\right)$. Equivalently, the differential form $\alpha=\sum_{j} y_{j} d x_{j}+\eta_{j} d \xi_{j}$ is closed. By Poincaré Lemma there exists $f \in \mathcal{C}_{M}^{\infty}$ such that $d f=\alpha$, equivalently $y_{j}=\left\{\xi_{j}, f\right\}$, and $\eta_{j}=\left\{f, x_{k}\right\}$.

There exists $B_{m+1} \in \mathcal{C}_{M}^{\infty}$ such that

$$
\exp \left(t \cdot a d\left(B_{m+1}\right)=\exp \left(a d\left(t^{m+1} f\right)\right) \circ \exp \left(t \cdot a d\left(B_{m}\right)\right)\right.
$$

and $B_{m+1}=B_{m} \bmod t^{m+1}$. The limit $B=\lim _{m \rightarrow \infty} B_{m}$ exists and satisfies the conclusions of the proposition. 
Proposition 6.2. Let $[\cdot, \cdot]$ be a deformation of a Poisson bracket $\{\cdot, \cdot\}$ on $M$ and $\mathcal{O}_{t}$ a t-adically complete submodule in $\mathcal{C}_{M}^{\infty}[[t]]$ being a commutative Lie subalgebra with respect to $[\cdot, \cdot \cdot]$. Let functions $x_{i} \in \mathcal{O}_{0}, \xi_{i} \in \mathcal{C}_{M}^{\infty}$ form Darboux coordinates with respect to $\{\cdot, \cdot\}$ on a contractible open set $U \subset M$. Then, there exist their lifts $\hat{x}_{i} \in \mathcal{O}_{t}, \hat{\xi}_{i} \in \mathcal{C}_{M}^{\infty}$ on $U$ which are Darboux coordinates with respect to $[\cdot, \cdot]$.

Proof. Since, by definition, $\mathcal{O}_{t} \rightarrow \sigma\left(\mathcal{O}_{t}\right)$ is surjective, we choose arbitrary lifts $\hat{x}_{j} \in \mathcal{O}_{t}$ of $x_{j}$. Note that $\hat{x}_{j}, \xi_{j}$ satisfy the both conclusions modulo $t$.

Let $\hat{x}_{j, 1}:=\hat{x}_{j}, \hat{\xi}_{j, 1}:=\xi_{j}$. Suppose that $m \geq 2$ and $\hat{x}_{j} \in \mathcal{O}_{t}, \hat{\xi}_{j, m} \in \mathcal{C}_{M}^{\infty}[[t]]$ satisfies the conclusion of the proposition modulo $t^{m+1}$. The assumption on $x_{j, m}, \xi_{j, m}$ implies that

$$
\begin{aligned}
{\left[\hat{x}_{j}, \hat{x}_{k}\right] } & =0 \\
{\left[\hat{\xi}_{j, m}, \hat{x}_{k}\right] } & =\delta_{j k}+y_{j k} t^{m+1} \bmod t^{m+2}, \\
{\left[\hat{\xi}_{j}, \hat{\xi}_{k}\right] } & =t^{m+1} z_{j k} \quad \bmod t^{m+2}
\end{aligned}
$$

for suitable $y_{j k}, z_{j k} \in \mathcal{C}_{M}^{\infty}$. The Jacobi identity and the commutation relations for $x_{j}, \xi_{j}$ imply that

$$
\begin{array}{r}
\left\{y_{j k}, x_{l}\right\}-\left\{y_{j l}, x_{k}\right\}=0, \\
\left\{z_{l j}, x_{k}\right\}+\left\{\xi_{j}, y_{l k}\right\}-\left\{\xi_{l}, y_{j k}\right\}=0, \\
\left\{z_{j k}, \xi_{l}\right\}+\left\{z_{k l}, \xi_{j}\right\}+\left\{z_{l j}, \xi_{k}\right\}=0 .
\end{array}
$$

These identities say that the differential form

$$
\alpha=y_{j k} d x_{j} \wedge d \xi_{k}+z_{j k} d x_{j} \wedge d x_{k}
$$

is closed.

By the Poincaré Lemma there exists a 1 -form $\beta=\sum a_{i} d \xi_{i}+\sum b_{i} d x_{i}$ on $U$ such that $d \beta=\alpha$.

Note that $\left\{x_{k}, a_{i}\right\}=0$ for all pairs $i, k$, since $\alpha$ contains no terms of the form $f d \xi_{i} \wedge d \xi_{k}$. Hence, $a_{i} \in \sigma\left(\mathcal{O}_{t}\right)$. Let $\hat{a}_{i}$ be a lift of $a_{i}$ in $\mathcal{O}_{t}$.

It is easy to check that $\hat{x}_{i, m+1}=x_{i, m}+t^{m+1} \hat{a}_{i}$ and $\hat{\xi}_{i, m+1}+t^{m+1} b_{i}$ satisfy the conclusions of the proposition modulo $t^{m+2}$. Hence, the limits $\hat{x}_{i}=\lim _{m \rightarrow \infty} \hat{x}_{i, m}, \hat{\xi}_{i}=\lim _{m \rightarrow \infty} \hat{\xi}_{i, m}$ exist and satisfy the conclusions of the proposition.

We will need a more strong assertion. 
Proposition 6.3. Let $[\cdot, \cdot]$ be a deformation of a Poisson bracket $\{\cdot, \cdot\}$ on $M$ and $\mathcal{O}_{t}$ a t-regular submodule in $\mathcal{C}_{M}^{\infty}[[t]]$, which is a commutative Lie subalgebra with respect to $[\cdot, \cdot]$. Let functions $x_{i} \in \mathcal{O}_{t}, \xi_{i} \in \mathcal{C}_{M}^{\infty}[[t]]$ form Darboux coordinates modulo $t^{k}, k>0$, with respect to $[\cdot, \cdot]$ on a contractible open set $U \subset M$. Then, there exist functions $a_{i} \in \mathcal{O}_{t}$, $b_{i} \in \mathcal{C}_{M}^{\infty}[[t]]$ on $U$ such that the functions $x_{i}+t^{k} a^{i}, \xi_{i}+t^{k} b_{i}$ form Darboux coordinates with respect to $[\cdot, \cdot]$.

Proof. The same as of Proposition 6.2.

\section{Deformation quantization on a polarized symplec- tic manifold}

\subsection{Deformation quantization}

Let us recall some definitions and facts about the deformation quantization on a smooth manifold $M$, see $[\mathrm{BFFLS}],[\mathrm{De}],[\mathrm{Fe}]$.

Definition 7.1. a) Let $\mathcal{C}_{M}^{\infty}$ be the sheaf of smooth complex valued functions on $M$. A formal deformation of $\mathcal{C}_{M}^{\infty}$ is a sheaf of $\mathbb{C}[[t]]$-algebras, $\mathbb{A}_{t}$, with an epimorphism $\sigma: \mathbb{A}_{t} \rightarrow$ $\mathcal{C}_{M}^{\infty}$ of $\mathbb{C}[[t]]$-algebras (called the symbol map) satisfying the condition: There exists an isomorphism of $\mathbb{C}[[t]]$-modules $\mathbb{A}_{t} \rightarrow \mathcal{C}_{M}^{\infty}[[t]]$ commuting with symbol maps. (Recall that the symbol map $\sigma: \mathcal{C}_{M}^{\infty}[[t]] \rightarrow \mathcal{C}_{M}^{\infty}$ takes $f_{0}+t f_{1}+\cdots$ to $f_{0}$.)

b) Two formal deformations $\mathbb{A}_{t 1}$ and $\mathbb{A}_{t 2}$ of $\mathcal{C}_{M}^{\infty}$ are equivalent if there exists a map of sheaves of $\mathbb{C}[[t]]$-algebras $\mathbb{A}_{t 1} \rightarrow \mathbb{A}_{t 2}$, commuting with symbol maps.

Suppose $\mathbb{A}_{t}$ is a formal deformation of $\mathcal{C}_{M}^{\infty}$. The formula

$$
\{a, b\}=\sigma\left(\frac{1}{t}[\widetilde{a}, \widetilde{b}]\right)
$$

where $a$ and $b$ are locally defined functions on $M$ and $\widetilde{a}, \widetilde{b}$ are their lifts with respect to $\sigma$, gives a well defined Poisson bracket on $\mathcal{C}_{M}^{\infty}$. It is clear that equivalent formal deformations have the same Poisson bracket.

Definition 7.2. A deformation quantization (DQ) on a symplectic manifold $(M, \omega)$ is a formal deformation of $\mathcal{C}_{M}^{\infty}$ whose Poisson bracket is equal to $\omega^{-1}$.

Definition 7.3. a) A star-product (SP) on $(M, \omega)$ is the structure of an associative algebra on the sheaf $\mathcal{C}_{M}^{\infty}[[t]]$ with the multiplication of the form

$$
f * g=\sum_{i \geq 0} t^{i} \mu_{i}(f, g), \quad f, g \in \mathcal{C}_{M}^{\infty},
$$


where all $\mu_{i}, i>0$, are bidifferential operators on $M$ vanishing on constants, i.e. $\mu_{i}(f, g)=$ 0 if $f$ or $g$ is a constant, $\mu_{0}(f, g)=f g$, and $\mu_{1}(f, g)-\mu_{1}(g, f)=\{f, g\}$, the Poisson bracket inverse to $\omega$.

b) Two star-products $\left(\mathcal{C}_{M}^{\infty}[[t]], \mu^{\prime}\right)$ and $\left(\mathcal{C}_{M}^{\infty}[[t]], \mu^{\prime \prime}\right)$ on $(M, \omega)$ are equivalent if there exists a power series $B=1+t B_{1}+\cdots$, where $B_{i}$ are differential operators vanishing on constants, such that $\mu^{\prime \prime}(f, g)=B \mu^{\prime}\left(B^{-1} f, B^{-1} g\right)$.

It is clear that any $\mathrm{SP}\left(\mathcal{C}_{M}^{\infty}[[t]], \mu\right)$ defines a DQ with the natural symbol map $\mathcal{C}_{M}^{\infty}[[t]] \rightarrow$ $\mathcal{C}_{M}^{\infty}, f_{0}+t f_{1}+\cdots \mapsto f_{0}$.

Proposition 7.4. The above assignment gives a one-to-one correspondence between the equivalence classes of SP's and DQ's.

Proof. Let $\mathbb{A}_{t}$ be a DQ. Let us prove that it is equivalent to a star-product. By definition of DQ, there exists an isomorphism of $\mathbb{C}[[t]]$-modules $\mathcal{C}_{M}^{\infty}[[t]] \rightarrow \mathbb{A}_{t}$ commuting with symbol maps. Let $\mu=\mu_{0}+t \mu_{1}+\cdots$ be the multiplication in the sheaf $\mathcal{C}_{M}^{\infty}[[t]]$ being the pullback of the multiplication in $\mathbb{A}_{t}$. In order to prove that each $\mu_{i}$ is a bidifferential operator it is enough to prove, according to the Peetre theorem, that $\operatorname{supp}(\mu(f, g)) \subset \operatorname{supp}(f) \cap \operatorname{supp}(g)$ for any functions $f$ and $g$ on $M$. But this is obvious because if, for example, $f=0$ on an open set $U$, then $\mu(f, g)=0$ on $U$, since $\mu$ is a map of sheaves. The same argument proves that two equivalent DQ's correspond to equivalent SP's.

Example 7.5. Moyal-Weyl star-product. Let $U$ be an open set in a symplectic manifold $(M, \omega)$, in which there exist Darboux coordinates, $x_{i}, y_{i}, i=1, \ldots, \frac{1}{2} \operatorname{dim} M$, so that $\omega=\sum_{i} d y_{i} \wedge d x_{i}$. Then, $\omega^{-1}=\sum_{i} \partial / \partial y_{i} \wedge \partial / \partial x_{i}$ and for $f, g \in C_{U}^{\infty}$ the multiplication formula

$$
f \otimes g \mapsto m \exp \left(\frac{t}{2} \sum_{i}\left(\partial / \partial y_{i} \otimes \partial / \partial x_{i}-\partial / \partial x_{i} \otimes \partial / \partial y_{i}\right)\right)(f \otimes g),
$$

where $m$ is the usual multiplication of functions, defines a star-product on $(U, \omega)$. This star-product is called the Moyal-Weyl star-product.

The following statement is well known and can be proven with the help of Hochschild and de Rham cohomology.

Proposition 7.6. Locally, any star-product on $(M, \omega)$ is equivalent to the Moyal-Weyl star-product. 


\subsection{Polarized deformation quantization}

Definition 7.7. a) A polarized deformation quantization (PDQ) on a polarized symplectic manifold $(M, \omega, \mathcal{P})$ is a pair $\left(\mathbb{A}_{t}, \mathbb{O}_{t}\right)$ where $\mathbb{A}_{t}$ is a DQ on $(M, \omega)$ and $\mathbb{O}_{t}$ is a $t$-adically complete commutative subalgebra in $\mathbb{A}_{t}$ such that $\sigma\left(\mathbb{O}_{t}\right)=\mathcal{O}_{\mathcal{P}}$, functions constant along $\mathcal{P}$.

b) Two PDQ's $\left(\mathbb{A}_{t 1}, \mathbb{O}_{t 1}\right)$ and $\left(\mathbb{A}_{t 2}, \mathbb{O}_{t 2}\right)$ of $(M, \omega, \mathcal{P})$ are equivalent if there exists an equivalence map of DQ's $\mathbb{A}_{t 1} \rightarrow \mathbb{A}_{t 2}$ which takes $\mathbb{O}_{t 1}$ to $\mathbb{O}_{t 2}$.

Since any DQ is equivalent to a $\mathrm{SP}$, any PDQ is equivalent to a triple $\left(\mathcal{C}_{M}^{\infty}[[t]], \mu_{t}, \mathcal{O}_{t}\right)$, where $\left(\mathcal{C}_{M}^{\infty}[[t]], \mu_{t}\right)$ is a SP and $\mathcal{O}_{t}$ is a commutative subalgebra in $\left(\mathcal{C}_{M}^{\infty}[[t]], \mu_{t}\right)$.

Proposition 7.8. Let $\left(\mathbb{A}_{t}, \mathbb{O}_{t}\right)$ be a $P D Q$. Then,

a) $\mathbb{O}_{t}$ is a maximal commutative subalgebra in $\mathbb{A}_{t}$.

b) $\mathbb{O}_{t}$ is a t-regular subalgebra in $\mathbb{A}_{t}$.

Proof. a) Locally, the bracket $[a, b]=\frac{1}{t}(a b-b a), a, b \in \mathbb{A}_{t}$, is a deformation of the Poisson bracket $\omega^{-1}$. So, the statement easily follows from Proposition 3.4.

b) Follows from a).

The following definition will play an auxiliary role in the paper.

Definition 7.9. A weakly polarized star-product (wPSP) on a polarized symplectic manifold $(M, \omega, \mathcal{P})$ is a triple $\left(\mathcal{C}_{M}^{\infty}[[t]], \mu_{t}, \mathcal{O}_{t}\right)$, where $\left(\mathcal{C}_{M}^{\infty}[[t]], \mu_{t}\right)$ is a $\mathrm{SP}, \mathcal{O}_{t}$ is a $t$-adically complete $\mathbb{C}[[t]]$-submodule in $\mathcal{C}_{M}^{\infty}[[t]]$ satisfying the conditions:

a) $\sigma\left(\mathcal{O}_{t}\right)=\mathcal{O}_{\mathcal{P}}$

b) $\mathcal{O}_{t}$ is a commutative subalgebra in $\mathcal{C}_{M}^{\infty}[[t]]$ with respect to the usual multiplication in $\mathcal{C}_{M}^{\infty}[[t]]$

c) $\mu_{t}$ being restricted to $\mathcal{O}_{t}$ coincides with the usual multiplication.

Definition 7.10. We say that a wPSP $\left(\mathcal{C}_{M}^{\infty}[[t]], \mu_{t}, \mathcal{O}_{t}\right)$ is a polarized star-product (PSP), if for any $a \in \mathcal{O}_{t}, b \in \mathcal{C}_{M}^{\infty}[[t]]$ the product $\mu_{t}(a, b)$ coincides with the usual product in $\mathcal{C}_{M}^{\infty}[[t]]$.

So, both wPSP and PSP are particular cases of a PDQ. We are going to prove that, in fact, any PDQ is equivalent to a PSP. But first we prove the following

Lemma 7.11. Any $P D Q$ is equivalent to a $w P S P$. 
Proof. Since any deformation quantization is equivalent to a star-product, it is enough to prove the following. Let $\mathcal{O}_{t}$ be a commutative subalgebra in a star-product $\left(\mathcal{C}_{M}^{\infty}[[t]], \mu_{t}\right)$ such that the triple $\left(\mathcal{C}_{M}^{\infty}[[t]], \mu, \mathcal{O}_{t}\right)$ forms a PDQ, in particular, $\mu_{t}(a, b)=\mu_{t}(b, a)$ for $a, b \in \mathcal{O}_{t}$. Then, there exists a differential operator $D_{t}=1+t D_{1}+\ldots$ on $M$ such that the multiplication $\widetilde{\mu}_{t}(f, g)=D_{t}^{-1} \mu\left(D_{t} f, D_{t} g\right)$ being restricted to $D_{t}^{-1} \mathcal{O}_{t}$ is the usual multiplication.

Suppose $\mu_{t}$ being restricted to $\mathcal{O}_{t}$ coincides with the usual multiplication modulo $t^{n}$. Then, $\mu(a, b)=a b+t^{n} \nu(a, b) \bmod t^{n}$ for $a, b \in \mathcal{O}_{t}$. It follows from Proposition $7.8 \mathrm{~b})$ that being considered modulo $t$, the bilinear form $\nu$ defines a Hochschild cocycle $\bar{\nu} \in \Gamma\left(M, \mathcal{D}^{2}\left(\mathcal{O}_{\mathcal{P}}, \mathcal{C}_{M}^{\infty}\right)\right)$ (see Proposition 2.5).

Since $\bar{\nu}$ is commutative, it follows from Proposition 2.5 that it is a coboundary. So, there exists a differential operator $\bar{D} \in \Gamma\left(M, \mathcal{D}^{1}\left(\mathcal{O}_{\mathcal{P}}, \mathcal{C}_{M}^{\infty}\right)\right)$ such that $d_{\text {Hoch }} \bar{D}=\bar{\nu}$.

Let $\widetilde{D}$ be a lift of $\bar{D}$ to a differential operator on $M$. Let $D_{n}=1+t^{n} \widetilde{D}$. It is easy to see that $D_{n}^{-1} \mathcal{O}_{t}$ is a commutative subalgebra in the star-product $\left(\mathcal{C}_{M}^{\infty}[[t]], \widetilde{\mu}_{t}\right)$ with $\widetilde{\mu}_{t}(a, b)=D_{n}^{-1} \mu_{t}\left(D_{n} a, D_{n} b\right)$, and $\widetilde{\mu}_{t}(a, b)=a b$ modulo $t^{n+1}$ for $a, b \in D_{n}^{-1} \mathcal{O}_{t}$.

By induction, we construct such differential operators $D_{n}$ for all $n$. Let $D=\prod_{n=1}^{\infty} D_{n}$, $\mathcal{O}_{t}^{\prime}=D^{-1} \mathcal{O}_{t}$, and $\mu_{t}^{\prime}(a, b)=D^{-1} \mu_{t}(D a, D b)$. Then $D$ gives an isomorphism between PDQ's $\left(\mathcal{C}_{M}^{\infty}[[t]], \mu_{t}, \mathcal{O}_{t}\right)$ and $\left(\mathcal{C}_{M}^{\infty}[[t]], \mu_{t}^{\prime}, \mathcal{O}_{t}^{\prime}\right)$, and the second triple is a wPSP, which proves the proposition.

Proposition 7.12. For any $w P S P\left(\mathcal{C}_{M}^{\infty}[[t]], \mu_{t}, \mathcal{O}_{t}\right)$, there exists a differential operator $D=1+t D_{1}+\cdots$ on $M$ such that $D f=f$ for $f \in \mathcal{O}_{t}$ and the multiplication $\mu_{t}^{\prime}(a, b)=$ $D^{-1} \mu_{t}(D a, D b)$ defines a PSP $\left(\mathcal{C}_{M}^{\infty}[[t]], \mu_{t}^{\prime}, \mathcal{O}_{t}\right)$.

Proof. It is obvious that $\mu=\mu_{t}$ defines a PSP modulo $t$. Proceeding by induction, we assume that there exists a wPSP multiplication $\mu^{\prime}$ equivalent to $\mu$ and being a PSP modulo $t^{n}$ with respect to $\mathcal{P}_{t}$. The proposition will be proved if we find a differential operator, $D_{n}$, such that $D_{n}(f)=0$ for all $f \in \mathcal{O}_{0}$ and the multiplication

$$
\mu^{\prime \prime}(a, b)=D^{-1} \mu^{\prime}(D a, D b)
$$

where $D=1+t^{n} D_{n}$, defines a PSP modulo $t^{n+1}$ with respect to $\mathcal{P}_{t}$.

Let

$$
\mu^{\prime}=\mu_{0}+t \mu_{1}^{\prime}+\cdots+t^{n-1} \mu_{n-1}^{\prime}+t^{n} \nu \quad \bmod t^{n+1} .
$$

By our assumption, elements $\mu_{1}^{\prime}, \ldots, \mu_{n-1}^{\prime}$ are strongly polarized and $\nu$ is polarized with respect to $\mathcal{O}_{t}$ (see the definition before Proposition 2.4). It follows from associativity of 
$\mu^{\prime}$ that

$$
d_{H o c h} \nu(a, b, c)=\sum_{i+j=n}\left(\mu_{i}^{\prime}\left(a, \mu_{j}^{\prime}(b, c)\right)-\mu_{i}^{\prime}\left(\mu_{j}^{\prime}(a, b), c\right)\right) .
$$

It is easy to check that each term in the right hand side is strongly polarized, since all $\mu_{i}^{\prime}$, $i=1, \ldots, n-1$, are such. So, $\nu$ satisfies the hypothesis of Proposition 2.4. Hence, there exists a polarized differential operator $D_{n}$ such that $\nu+d_{H o c h} D_{n}$ is a strongly polarized bidifferential operator. It is obvious that the multiplication $\mu^{\prime \prime}$ defined in (7.4) with $D_{n}$ just constructed is as required.

Corollary 7.13. Any PDQ is equivalent to a PSP.

Proof. Follows from Lemma 7.11 and Proposition 7.12.

Proposition 7.14. a) Let $\left(\mathcal{C}_{M}^{\infty}[[t]], \mu_{t}, \mathcal{O}_{t}\right)$ be a PSP on $(M, \omega, \mathcal{P})$. Let $\mathcal{P}_{t}=\left(d \mathcal{O}_{t}\right)^{\perp}$. Then, $\mathcal{P}_{t}$ is a deformation of $\mathcal{P}$ and $\mathcal{O}_{t}=\mathcal{O}_{\mathcal{P}_{t}}$.

b) Let $\left(\mathcal{C}_{M}^{\infty}[[t]], \widetilde{\mu}_{t}, \widetilde{\mathcal{O}}_{t}\right)$ be another PSP on $(M, \omega, \mathcal{P})$ equivalent to $\left(\mathcal{C}_{M}^{\infty}[[t]], \mu_{t}, \mathcal{O}_{t}\right)$ as a $P D Q$. Then, there exists a formal automorphism of $M$ which takes $\mathcal{P}_{t}$ to $\widetilde{\mathcal{P}}_{t}=\left(d \widetilde{\mathcal{O}}_{t}\right)^{\perp}$.

c) Let $\left(\mathcal{C}_{M}^{\infty}[[t]], \mu_{t}, \mathcal{O}_{t}\right)$ and $\left(\mathcal{C}_{M}^{\infty}[[t]], \widetilde{\mu}_{t}, \mathcal{O}_{t}\right)$ are two equivalent PSP's with the same $\mathcal{O}_{t}$. Let $D=1+t D_{1}+\cdots$ gives an equivalence. Then, there exists a decomposition, $D=D^{\prime} e^{t X}$, where $D^{\prime}$ is a differential operator identical on $\mathcal{O}_{t}$ and $X$ is a formal vector field on $M$ taking $\mathcal{O}_{t}$ to itself.

Proof. a) follows from Proposition 4.4.

b). Let us put $X_{0}=0$. Then the automorphism $e^{t X_{0}}=I d$ takes $\mathcal{P}_{t}$ to $\widetilde{\mathcal{P}}_{t}$ modulo $t$. Suppose we have constructed a formal vector field $X_{k}$ such that the formal automorphism $e^{t X_{k-1}}$ of $M$ takes $\mathcal{P}_{t}$ to $\widetilde{\mathcal{P}}_{t}$ modulo $t^{k}$. Then, replacing $\mathcal{P}_{t}$ by $e^{t X_{k-1}} \mathcal{P}_{t}$ we may assume that $\mathcal{P}_{t}$ and $\widetilde{\mathcal{P}}_{t}$ coincide modulo $t^{k}$. The proposition will be proved, if we show that it is possible to find a vector field $Y$ such that $e^{t^{k} Y}$ takes $\mathcal{P}_{t}$ to $\widetilde{\mathcal{P}}_{t}$ modulo $t^{k+1}$.

Since, by our assumption, the SP's $\left(\mathcal{C}_{M}^{\infty}[[t]], \mu_{t}\right)$ and $\left(\mathcal{C}_{M}^{\infty}[[t]], \widetilde{\mu}_{t}\right)$ are equivalent and coincide modulo $t^{k}$, there exists a differential operator $1+t^{k} D_{k}$ realizing that equivalence. Since $1+t^{k} D_{k}$ takes $\mathcal{O}_{t}$ to $\widetilde{\mathcal{O}}_{t}$ and on the both of these subalgebras the respecting multiplications $\mu_{t}$ and $\widetilde{\mu}_{t}$ are trivial, $D_{k}$ being restricted to $\mathcal{O}_{0}$ is a derivation from $\mathcal{O}_{0}$ to $\mathcal{C}_{M}^{\infty}$. Let $Y$ be an extension of that restricted $D_{k}$ to a derivation on $\mathcal{C}_{M}^{\infty}$. It is clear that $e^{t^{k} Y}$ takes $\mathcal{P}_{t}$ to $\widetilde{\mathcal{P}}_{t}$ modulo $t^{k+1}$.

c). The operator $D$ being restricted to $\mathcal{O}_{t}$ is a formal automorphism of $\mathcal{O}_{t}$. Since formal automorphisms form a pro-unipotent group, there exists $X^{\prime} \in \operatorname{Der}\left(\mathcal{O}_{t}\right)$ such that 
$D$ being restricted to $\mathcal{O}_{t}$ is equal to $e^{t X^{\prime}}$. Let $X \in \operatorname{Der}\left(\mathcal{C}_{M}^{\infty}\right)$ be a lift of $X^{\prime}$. We put $D^{\prime}=D e^{-t X}$ which is obviously identical on $\mathcal{O}_{t}$.

Example 7.15. Moyal-Wick PSP. Let $(M, \omega, \mathcal{P})$ be a polarized symplectic manifold. Let $U$ be an open set in $M$ where there exist Darboux coordinates, $x_{i}, y_{i},\left(d x_{i}\right)^{\perp}=\mathcal{P}$, $i=1, \ldots, \frac{1}{2} \operatorname{dim} M$, so that $\omega=\sum_{i} d y_{i} \wedge d x_{i}$. Then, $\omega^{-1}=\sum_{i} \partial / \partial y_{i} \wedge \partial / \partial x_{i}$ and for $f, g \in C_{U}^{\infty}$ the multiplication formula

$$
f \otimes g \mapsto m \exp \left(t \sum_{i} \partial / \partial y_{i} \otimes \partial / \partial x_{i}\right)(f \otimes g),
$$

where $m$ is the usual multiplication of functions, defines a PSP on $(U, \omega, \mathcal{P})$. This PSP is called the Moyal-Wick polarized star-product.

Note that the functions $a_{i}, f_{i}$ satisfying the Darboux relations with respect to the Poisson bracket $\omega^{-1}$ also satisfy the Darboux relations with respect to the deformed bracket $\frac{1}{t}[\cdot, \cdot]$, where $[\cdot, \cdot]$ is the commutator of the Moyal-Wick PSP.

Let us remark that the Moyal-Weyl SP from Example 7.5 constructed using the same Darboux coordinates gives just a wPSP but not a PSP.

Proposition 7.16. Locally, any PSP on $(M, \omega, \mathcal{P})$ is equivalent to the Moyal-Wick PSP.

Proof. Let us prove that any two PSP's are locally equivalent. Since any SP's are locally equivalent, we may suppose that there are given two PSP's, $\left(\mathcal{C}_{M}^{\infty}[[t]], \mu_{t}, \mathcal{O}_{t}\right)$ and $\left(\mathcal{C}_{M}^{\infty}[[t]], \mu_{t}, \widetilde{\mathcal{O}}_{t}\right)$, with the same multiplication and different polarizations, and we have to prove that they are, locally, equivalent. Let $x_{i}, y_{i}$ are Darboux coordinates with respect to $\omega$ such that $\left(d x_{i}\right)^{\perp}=\mathcal{P}$. By Proposition 6.2, there exist their lifts $x_{i t}, y_{i t}$ and $x_{i t}^{\prime}, y_{i t}^{\prime}$ which satisfy the Darboux relations with respect to the bracket $[a, b]=\frac{1}{t}\left(\mu_{t}(a, b)-\mu_{t}(b, a)\right)$, and

$x_{i t} \in \mathcal{O}_{t}, x_{i t}^{\prime} \in \widetilde{\mathcal{O}}_{t}$. By Proposition 6.1, there exists, locally, an inner automorphism of the SP $\left(\mathcal{C}_{M}^{\infty}[[t]], \mu_{t}\right)$ that takes $x_{i t}, y_{i t}$ to $x_{i t}^{\prime}, y_{i t}^{\prime}$. It follows that this automorphism takes $\mathcal{O}_{t}$ to $\widetilde{\mathcal{O}}_{t}$.

\section{Characteristic classes of PDQ's and PSP's}

\subsection{Extension class associated with a PDQ}

Let $\left(\mathbb{A}_{t}, \mathbb{O}_{t}\right)$ be a PDQ on a polarized symplectic manifold $(M, \omega, \mathcal{P})$. Since any PDQ is equivalent to a PSP, the sheaf $\mathbb{O}_{t}$ is isomorphic to $\mathcal{O}_{\mathcal{P}_{t}}$ for some deformed distribution $\mathcal{P}_{t}$. Thus, the sheaves $\operatorname{Der}\left(\mathbb{O}_{t}\right)$ and $\Omega_{\mathbb{O}_{t}}^{1 c l}$ are well defined (see Section 2). Let

$$
F\left(\mathbb{A}_{t}, \mathbb{O}_{t}\right)=\left\{b \in \mathbb{A}_{t} ;\left[b, \mathbb{O}_{t}\right] \subset \mathbb{O}_{t}\right\},
$$


where $[\cdot, \cdot]$ denotes the commutator in $\mathbb{A}_{t}$. It is clear that $F\left(\mathbb{A}_{t}, \mathbb{O}_{t}\right)$ is a sheaf of Lie algebras with the bracket $\frac{1}{t}[\cdot, \cdot]$ and the center $\mathbb{O}_{t}$. Moreover, any element $b \in F\left(\mathbb{A}_{t}, \mathbb{O}_{t}\right)$ determines the derivation $\frac{1}{t}[b, \cdot]$ of $\mathbb{O}_{t}$ and, due to Proposition 6.2, this correspondence defines an epimorphism $\sigma: F\left(\mathbb{A}_{t}, \mathbb{O}_{t}\right) \rightarrow \operatorname{Der}\left(\mathbb{O}_{t}\right)$.

We consider $F\left(\mathbb{A}_{t}, \mathbb{O}_{t}\right)$ as a left $\mathbb{O}_{t}$-module with respect to multiplication in $\mathbb{A}_{t}$. As a Lie algebra sheaf, $F\left(\mathbb{A}_{t}, \mathbb{O}_{t}\right)$ is an extension of $\operatorname{Der}\left(\mathbb{O}_{t}\right)$.

So, we have the following exact sequence of Lie algebras and $\mathcal{O}_{t}$-modules:

$$
0 \longrightarrow \mathbb{O}_{t} \stackrel{\iota}{\longrightarrow} F\left(\mathbb{A}_{t}, \mathbb{O}_{t}\right) \stackrel{\sigma}{\longrightarrow} \operatorname{Der}\left(\mathbb{O}_{t}\right) \longrightarrow 0 \text {. }
$$

According to the terminology of $[\overline{\mathrm{BB}}],[\mathrm{BK}], F\left(\mathbb{A}_{t}, \mathbb{O}_{t}\right)$ is called $a \mathbb{O}_{t}$-extension of $\operatorname{Der}\left(\mathbb{O}_{t}\right)$.

We say that a map of Lie algebras and $\mathbb{O}_{t}$-modules, $s: \operatorname{Der}\left(\mathbb{O}_{t}\right) \rightarrow F\left(\mathbb{A}_{t}\right)$, given over an open set of $M$ is a splitting of (8.2), if $s \sigma=i d$. Since $\left(\mathbb{A}_{t}, \mathbb{O}_{t}\right)$ can be realized as a PSP and, locally, there exist Darboux coordinates with respect to $\frac{1}{t}[\cdot, \cdot]$, the sequence (8.2) locally splits (see the next subsection, where splittings are presented with the help of Darboux coordinates).

Lemma 8.1. Let $s$ and $s^{\prime}$ are two splittings of (8.9) over an open set of $M$. Then, $s-s^{\prime} \in \Omega_{\mathbb{O}_{t}}^{1 c l}$.

Proof. Direct calculation.

Let us define the extension class of (8.1) in the following way. Let $\left\{U_{\alpha}\right\}$ be an open covering of $M$ such that over each $U_{\alpha}$ there is a splitting, $s_{\alpha}$, of $F\left(\mathbb{A}_{t}, \mathbb{O}_{t}\right)$. By Lemma 8.1, $f_{\alpha, \beta}=s_{\beta}-s_{\alpha}$ is a section of $\Omega_{\mathbb{O}_{t}}^{1 c l}$ over $U_{\alpha} \cap U_{\beta}$. We define $c l_{E x t}\left(F\left(\mathbb{A}_{t}, \mathbb{O}_{t}\right)\right)$ as the element of $H^{1}\left(M, \Omega_{\mathbb{O}_{t}}^{1 c l}\right)$ represented by the collection $\left\{f_{\alpha, \beta}\right\}$. One can prove that given $\mathbb{O}_{t}$, the extension class $c l_{E x t}\left(F\left(\mathbb{A}_{t}, \mathbb{O}_{t}\right)\right)$ determines a $\mathbb{O}_{t}$-extension of $\operatorname{Der}\left(\mathbb{O}_{t}\right)$ up to equivalence.

We will denote the element $c l_{E x t}\left(F\left(\mathbb{A}_{t}, \mathbb{O}_{t}\right)\right)$ by $c l_{E x t}\left(\mathbb{A}_{t}, \mathbb{O}_{t}\right)$ and call it the extension class of the $\operatorname{PDQ}\left(\mathbb{A}_{t}, \mathbb{O}_{t}\right)$.

In the next subsection, the extension class of a PSP, $\left(\mathcal{C}_{M}^{\infty}[[t]], \mu_{t}, \mathcal{O}_{t}\right)$, will be represented as an element of $\Gamma\left(M, d \mathcal{P}_{t}^{\perp}\right) / d\left(\Gamma\left(M, \mathcal{P}_{t}^{\perp}\right)\right), \mathcal{P}_{t}=\mathcal{P}_{\mathcal{O}_{t}}$, with the help of a characteristic 2form associated with that PSP.

\subsection{Characteristic 2-form associated with a PSP}

Let $\left(\mathcal{C}_{M}^{\infty}[[t]], \mu_{t}, \mathcal{O}_{t}\right)$ be a PSP that we denote for shortness by $\left(\mu_{t}, \mathcal{O}_{t}\right)$. We denote $\mathcal{P}_{t}=$ $\mathcal{P}_{\mathcal{O}_{t}}$. Then the extension $F\left(\mu_{t}, \mathcal{O}_{t}\right)$ coincides as a left $\mathcal{O}_{t}$-module with a $\mathcal{O}_{t}$-submodule of $\mathcal{C}_{M}^{\infty}[[t]]$ with respect to the usual multiplication in $\mathcal{C}_{M}^{\infty}[[t]]$. In this case, the local splittings 
of $F\left(\mu_{t}, \mathcal{O}_{t}\right)$ are differential forms of $\operatorname{Hom}_{\mathcal{O}_{t}}\left(\operatorname{Der}\left(\mathcal{O}_{t}\right), \mathcal{C}_{M}^{\infty}[[t]]\right)=\mathcal{P}^{\perp}$. These forms can be described explicitly by Darboux coordinates.

Let $\left\{U_{\alpha}\right\}$ be an open covering of $M$ such that each $U_{\alpha}$ has Darboux coordinates $x_{\alpha i}$, $y_{\alpha i}, x_{\alpha i} \in \mathcal{O}_{t}, i=1, \ldots, n$, with respect to the bracket $[a, b]=\frac{1}{t}\left(\mu_{t}(a, b)-\mu_{t}(b, a)\right)$ (in particular, $\left.\left[y_{\alpha i}, x_{\alpha j}\right]=\delta_{i j}\right)$. By Proposition 6.2 such a covering exists. Then, on $U_{\alpha}$, the

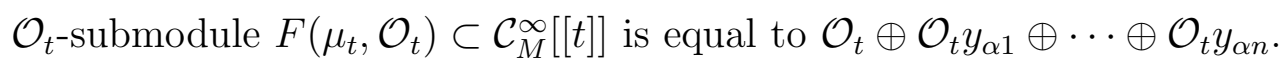

Splittings $s_{\alpha}$ may be taken by the condition $x_{\alpha i} \mapsto y_{\alpha i}$, and the corresponding forms are

$$
s_{\alpha}=\sum_{i} y_{\alpha i} d x_{\alpha i}
$$

By Lemma 8.1, $d s_{\alpha}=d s_{\beta}$ on $U_{\alpha} \cap U_{\beta}$. So, forms $s_{\alpha}$ define the global 2-form

$$
\omega_{t} \in \omega_{0}+t \Gamma\left(M, d \mathcal{P}_{t}^{\perp}\right), \quad \omega_{t}=d s_{\alpha}=\sum_{i} d y_{\alpha i} \wedge d x_{\alpha i}
$$

Lemma 8.2. This form represents the extension class $c_{E x t}\left(\mu_{t}, \mathcal{O}_{t}\right)$ by the isomorphism (2.8).

Proof. Clear.

Lemma 8.1 shows that if we take other splittings of $F\left(\mu_{t}, \mathcal{O}_{t}\right)$, the procedure above gives the same form $\omega_{t}$. So, in the case of PSP, it is well defined not only the class $c l_{E x t}\left(\mu_{t}, \mathcal{O}_{t}\right)$ but also its representative in $\omega_{0}+t \Gamma\left(M, d \mathcal{P}_{t}^{\perp}\right)$ that we call the characteristic 2 -form of the PSP and denote it by $c_{P S P}\left(\mu_{t}, \mathcal{O}_{t}\right)$.

Given a SP, $\left(\mathcal{C}_{M}^{\infty}[[t]], \mu_{t}\right)$, let us denote $[a, b]_{\mu_{t}}=\frac{1}{t}\left(\mu_{t}(a, b)-\mu_{t}(b, a)\right), a, b \in \mathcal{C}_{M}^{\infty}[[t]]$. The bracket [, $]_{\mu_{t}}$ is a deformation of the initial Poisson bracket on $M$.

Proposition 8.3. Let $\left(\mu_{t}, \mathcal{O}_{t}\right),\left(\widetilde{\mu}_{t}, \mathcal{O}_{t}\right)$ be PSP's with the same $\mathcal{O}_{t}$. Then, $\operatorname{cl}_{P S P}\left(\mu_{t}, \mathcal{O}_{t}\right)=$ $\operatorname{cl}_{P S P}\left(\widetilde{\mu}_{t}, \mathcal{O}_{t}\right)$ if and only if $[,]_{\mu_{t}}=[,]_{\tilde{\mu}_{t}}$.

Proof. If $c l_{P S P}\left(\mu_{t}, \mathcal{O}_{t}\right)=c l_{P S P}\left(\widetilde{\mu}_{t}, \mathcal{O}_{t}\right)$, then forms (8.3) can be taken to be the same. This implies that there exist common Darboux coordinates with respect to $[,]_{\mu_{t}}$ and $[,]_{\tilde{\mu}_{t}}$.

Proposition 8.4. Let $\left(\mu_{t}, \mathcal{O}_{t}\right),\left(\widetilde{\mu}_{t}, \mathcal{O}_{t}\right)$ be two PSP's with the same $\mathcal{O}_{t}$. Let $\mathcal{P}_{t}=\mathcal{P}_{\mathcal{O}_{t}}$ and $\Gamma \mathcal{P}_{t}$ denote the module of global sections of $\mathcal{P}_{t}$. Then, the 2-forms $\operatorname{cl}_{P S P}\left(\mu_{t}, \mathcal{O}_{t}\right)$, $c_{P S P}\left(\widetilde{\mu}_{t}, \mathcal{O}_{t}\right)$ are lying on the same orbit of $e^{t \Gamma \mathcal{P}_{t}}$ if and only if there exists a formal differential operator $D=1+t D_{1}+\cdots$ identical on $\mathcal{O}_{t}$ such that $\widetilde{\mu}_{t}(a, b)=D^{-1} \mu_{t}(D a, D b)$. 
Proof. Let us denote $\omega_{t}=c l_{P S P}\left(\mu_{t}, \mathcal{O}_{t}\right), \widetilde{\omega}_{t}=c l_{P S P}\left(\widetilde{\mu}_{t}, \mathcal{O}_{t}\right)$. Assume such $D$ exists. Let $s_{\alpha}$ be forms from (8.3) corresponding to $\left(\mu_{t}, \mathcal{O}_{t}\right)$. Let $\widetilde{s}_{\alpha}$ be forms obtained from $s_{\alpha}$ by applying $D$. Lemmas 8.1 and 2.7 imply that there exist $f_{\alpha, \beta} \in \mathcal{O}_{t}$ over $U_{\alpha} \cap U_{\beta}$ such that $s_{\alpha}-s_{\beta}=d f_{\alpha, \beta}$. Since $D$ acts on $\mathcal{O}_{t}$ trivially, one has $\widetilde{s}_{\alpha}-\widetilde{s}_{\beta}=d f_{\alpha, \beta}$, too. This means that $\widetilde{s}_{\alpha}-s_{\alpha}$ does not depend on $\alpha$ and give a global form, $b$, of $\mathcal{P}_{t}^{\perp}$. Since $\omega_{t}=d s_{\alpha}$, $\widetilde{\omega}_{t}=d \widetilde{s}_{\alpha}$, one has $\widetilde{\omega}_{t}=\omega_{t}+d b$. By Lemma 4.6, there exists a formal automorphism, $e^{t Y}$, $Y \in \Gamma \mathcal{P}_{t}$, taking $\omega_{t}$ to $\widetilde{\omega}_{t}$.

Conversely, let us suppose that $\omega_{t}$ and $\widetilde{\omega}_{t}$ are on the same orbit of $e^{t \Gamma \mathcal{P}_{t}}$. Assume that we have found a differential operator identical on $\mathcal{O}_{t}$ which transforms $\widetilde{\mu}_{t}$ to a multiplication $\widetilde{\mu}_{t}^{\prime}$ that is equal to $\mu_{t}$ modulo $t^{k}$, i.e.

$$
\widetilde{\mu}_{t}^{\prime}-\mu_{t}=t^{k} \nu+\cdots \text {. }
$$

By the previous part of the proof, the corresponding form $\widetilde{\omega}_{t}^{\prime}$ is also lying on the same orbit as $\omega_{t}$. The proposition will be proved if we find a differential operator $1+t^{k} D_{k}$, $D_{k}\left(\mathcal{O}_{t}\right)=0$, which transforms $\widetilde{\mu}_{t}^{\prime}$ to $\mu_{t}$ modulo $t^{k+1}$. Let us prove that.

Since $\widetilde{\mu}_{t}^{\prime}=\mu_{t}$ mod $t^{k}$, we can choose, by Proposition 6.3, systems of Darboux coordinates with respect to $[,]_{\widetilde{\mu}_{t}^{\prime}}$ and $[,]_{\mu_{t}}$ that coincides modulo $t^{k}$. It follows that $\widetilde{\omega}_{t}^{\prime}=\omega_{t} \bmod$ $t^{k}$. Hence, there is a formal automorphism $e^{t^{k} X}, X \in \mathcal{P}_{t}$, which takes $\widetilde{\omega}_{t}^{\prime}$ to $\omega_{t}$. Applying $e^{t^{k} X}$ to $\widetilde{\mu}_{t}^{\prime}$, we obtain a multiplication that is still equal to $\mu_{t}$ modulo $t^{k}$ but whose characteristic form is equal to $\omega_{t}$. So, we come to the situation when the multiplications $\widetilde{\mu}_{t}^{\prime}$ and $\mu_{t}$ have the same characteristic form $\omega_{t}$.

By Proposition 8.3, $\widetilde{\mu}_{t}^{\prime}$ and $\mu_{t}$ have the same commutator. This implies that bidifferential operator $\nu$ in (8.5) is commutative. Moreover, it is a Hochschild strongly polarized cocycle (see Section 2). So, there exists a polarized differential operator $D_{k}$ such that $d_{H o c h} D=\nu$. It follows that transformation $1+t^{k} D_{k}$ applying to $\widetilde{\mu}_{t}^{\prime}$ gives a multiplication equal to $\mu_{t}$ modulo $t^{k+1}$ and identical on $\mathcal{O}_{t}$.

\subsection{Characteristic pairs for PSP's and PDQ's}

Let $(M, \omega, \mathcal{P})$ be a polarized symplectic manifold. Let us denote by $\operatorname{Aut}(M)$ the group of formal automorphisms of $M$ and by $\mathcal{Y}=\mathcal{Y}(M, \omega, \mathcal{P})$ the set of pairs $\left(\omega_{t}, \mathcal{P}_{t}\right)$, where $\omega_{t}=\omega_{0}+t \omega_{1}+\cdots$ is a formal symplectic form being a deformation of $\omega=\omega_{0}$ and $\mathcal{P}_{t}$ is a polarization of $\omega_{t}$ being a deformation of $\mathcal{P}$. The group $\operatorname{Aut}(M)$ naturally acts on $\mathcal{Y}$.

It is natural to assign to a PSP $\left(\mu_{t}, \mathcal{O}_{t}\right)$ on $(M, \omega, \mathcal{P})$ a pair $\left(\omega_{t}, \mathcal{P}_{t}\right) \in \mathcal{Y}$, where $\omega_{t}=c_{P S P}\left(\mu_{t}, \mathcal{O}_{t}\right)$ and $\mathcal{P}_{t}=\mathcal{P}_{\mathcal{O}_{t}}$. So, we obtain the map

$$
\tau:\{\mathrm{PSP} ' \mathrm{~s}\} \rightarrow \mathcal{Y}
$$


Proposition 8.5. Two PSP's $\left(\mu_{t}, \mathcal{O}_{t}\right)$ and $\left(\widetilde{\mu}_{t}, \widetilde{\mathcal{O}}_{t}\right)$ are equivalent if and only if $\tau\left(\mu_{t}, \mathcal{O}_{t}\right)$ and $\tau\left(\widetilde{\mu}_{t}, \widetilde{\mathcal{O}}_{t}\right)$ are lying on the same orbit in $\mathcal{Y}$ under the Aut $(M)$-action.

Proof. Let $\left(\mu_{t}, \mathcal{O}_{t}\right)$ and $\left(\widetilde{\mu}_{t}, \widetilde{\mathcal{O}}_{t}\right)$ be equivalent. Let us prove that the pairs $\tau\left(\mu_{t}, \mathcal{O}_{t}\right)$ and $\tau\left(\widetilde{\mu}_{t}, \widetilde{\mathcal{O}}_{t}\right)$ are lying on the same orbit. By Proposition $\left.7.14 \mathrm{~b}\right)$, c) one can find a formal automorphism of $M$ such that after its applying we come to the situation when $\left(\widetilde{\mu}_{t}, \widetilde{\mathcal{O}}_{t}\right)$ turns into a PSP, $\left(\widetilde{\mu}_{t}, \mathcal{O}_{t}\right)$, with the same $\mathcal{O}_{t}$ as in $\left(\mu_{t}, \mathcal{O}_{t}\right)$ and the equivalence morphism from $\left(\mu_{t}, \mathcal{O}_{t}\right)$ to $\left(\widetilde{\mu}_{t}, \mathcal{O}_{t}\right)$ is given by a differential operator identical on $\mathcal{O}_{t}$. Now the statement follows from Proposition 8.4.

Conversely, suppose that for $\left(\mu_{t}, \mathcal{O}_{t}\right)$ and $\left(\widetilde{\mu}_{t}, \widetilde{\mathcal{O}}_{t}\right)$ the corresponding pairs $\left(\omega_{t}, \mathcal{P}_{t}\right)$, $\left(\widetilde{\omega}_{t}, \widetilde{\mathcal{P}}_{t}\right)$ lie on the same orbit. Let us prove that those PSP's are equivalent. Let $B$ be a formal automorphism of $M$ sending $\widetilde{\mathcal{P}}_{t}$ to $\mathcal{P}_{t}$. Applying $B$ to $\left(\widetilde{\mu}_{t}, \widetilde{\mathcal{O}}_{t}\right)$, we come to the case when $\widetilde{\mathcal{P}}_{t}=\mathcal{P}_{t}$. So, we may suppose that $\left(\widetilde{\omega}_{t}, \widetilde{\mathcal{P}}_{t}\right)=\left(\widetilde{\omega}_{t}, \mathcal{P}_{t}\right)$. Since the pairs $\left(\omega_{t}, \mathcal{P}_{t}\right)$, $\left(\widetilde{\omega}_{t}, \mathcal{P}_{t}\right)$ lie on the same orbit, there exists $X \in \Gamma \mathcal{P}_{t}$ such that $\widetilde{\omega}_{t}=e^{t X} \omega_{t}$. Now the statement follows from Proposition 8.4.

Let us denote by $[\mathcal{Y}]$ the set of orbits in $\mathcal{Y}=\mathcal{Y}(M, \omega, \mathcal{P})$.

Corollary 8.6. The map $\tau$ induces the embedding

$$
\bar{\tau}:\left\{\text { classes of } P D Q^{\prime} s\right\} \rightarrow[\mathcal{Y}]
$$

Proof. Let $\left(\mathbb{A}_{t}, \mathbb{O}_{t}\right)$ be a PDQ on $(M, \omega, \mathcal{P})$. Then, by Corollary 7.13, there exists a PSP, $\left(\mu_{t}, \mathcal{O}_{t}\right)$, equivalent to $\left(\mathbb{A}_{t}, \mathbb{O}_{t}\right)$. We put $\bar{\tau}\left(\mathbb{A}_{t}, \mathbb{O}_{t}\right)=\left[\tau\left(\mu_{t}, \mathcal{O}_{t}\right)\right]$, the orbit passing through the pair $\tau\left(\mu_{t}, \mathcal{O}_{t}\right)$. By Propositions 7.14 and 8.5, this map is correctly defined, i.e. does not depends on the choice of an equivalent PSP. Proposition 8.5 also shows that being descended to equivalence classes of PDQ's $\bar{\tau}$ is embedding.

In the next section we will prove that any element of $\mathcal{Y}$ is equal to $\tau\left(\mu_{t}, \mathcal{O}_{t}\right)$ for a PSP $\left(\mu_{t}, \mathcal{O}_{t}\right)$, which implies that the map $\bar{\tau}$ is, in fact, an isomorphism.

\section{Existence of polarized deformation quantizations and relation between the extension and Fedosov classes of a PDQ}

Let $\left(M, \omega_{0}\right)$ be a symplectic manifold. It is known that all equivalence classes of deformation quantizations (DQ) on $M$ with the Poisson bracket $\omega_{0}^{-1}$ can be obtained by the Fedosov method. According to this method, starting with a symplectic connection, one 
constructs a flat connection, $D$, (called the Fedosov connection) in the Weyl algebra defined on the cotangent bundle to $M$ via the Poisson bracket $\omega_{0}^{-1}$. The quantized algebra, $\mathbb{A}_{t}$, is realized as the subalgebra of flat sections in the Weyl algebra. The Weyl curvature of $D$ (see below), being a closed scalar 2-form of the form $\theta_{t}=\omega_{0}+t \omega_{1}+\cdots$, defines the Fedosov class

$$
c l_{F}\left(\mathbb{A}_{t}\right)=\left[\theta_{t}\right] \in\left[\omega_{0}\right]+t H^{2}(M, \mathbb{C}[[t]])
$$

It is also known that the correspondence $\mathbb{A}_{t} \mapsto c l_{F}\left(\mathbb{A}_{t}\right)$ is a bijection between the set of

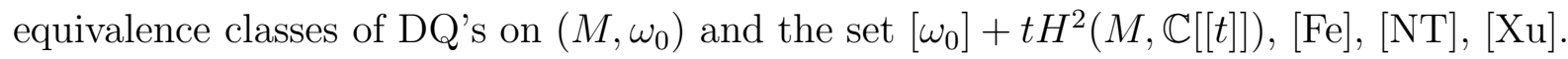

Let $(M, \omega, \mathcal{P})$ be a polarized symplectic manifold and $\left(\omega_{t}, \mathcal{P}_{t}\right) \in \mathcal{Y}(M, \omega, \mathcal{P})$ a deformation of the pair $(\omega, \mathcal{P})$, see Subsection 8.3. We adapt the Fedosov method to construct a PSP, $\left(\mu_{t}, \mathcal{O}_{t}\right)$, such that $\tau\left(\mu_{t}, \mathcal{O}_{t}\right)=\left(\omega_{t}, \mathcal{P}_{t}\right)$. We start with a $\mathcal{P}_{t^{-}}$symplectic connection, $\nabla$, and construct the Fedosov connection on the same Weyl algebra. We will see that by realizing the Fedosov scheme in presence of a polarization, the form $\omega_{t}$ appears as a so-called Wick curvature of the Fedosov connection. Moreover, $\omega_{t}$ differs from the Weyl curvature of that Fedosov connection by the form $\frac{t}{2} \operatorname{tr}\left(\left.\nabla^{2}\right|_{\mathcal{P}_{t}}\right)$.

\subsection{Some notations}

Let $\mathcal{E}$ be a formal vector bundle over $M$, i.e. a free $\mathcal{C}_{M}^{\infty}[[t]]$-module of finite rank over $M$. Denote by $T^{k}(\mathcal{E})$ the $k$-th tensor power of $\mathcal{E}$ over $\mathcal{C}_{M}^{\infty}[[t]]$ and by $T(\mathcal{E})$ the corresponding tensor algebra completed in the $\{\mathcal{E}, t\}$-adic topology. Similarly we define the completed symmetric algebra $S(\mathcal{E})$. For a subbundle $\mathcal{P}$ of $\mathcal{E}$, we denote by $\operatorname{sym}_{\mathcal{P}}: S(\mathcal{P}) \rightarrow T(\mathcal{E})$ the natural embedding of $\mathcal{C}_{M}^{\infty}[[t]]$-modules defined by symmetrization.

Let $\Lambda(\mathcal{E})$ be the exterior algebra of $\mathcal{E}$ over $\mathcal{C}_{M}^{\infty}[[t]]$. We will consider $T(\mathcal{E}) \otimes \Lambda(\mathcal{E})$ as a graded super-algebra regarding a section $x \in T(\mathcal{E}) \otimes \Lambda^{k}(\mathcal{E})$ of degree $k$ even (odd) if $k$ is even (odd).

Denote by $\delta_{T(\mathcal{E})}$ the continuous $\mathcal{C}_{M}^{\infty}[[t]]$-linear derivation of $T(\mathcal{E}) \otimes \Lambda(\mathcal{E})$ defined by the $\operatorname{map} T^{1}(\mathcal{E}) \otimes 1 \rightarrow 1 \otimes \Lambda^{1}(\mathcal{E}), v \otimes 1 \mapsto 1 \otimes v, v \in \mathcal{E}$ is a section. It is clear that $\delta_{T(\mathcal{E})}$ is a derivation of degree 1 and $\delta_{T(\mathcal{E})}^{2}=0$. It is easy to see that for any subbundle $\mathcal{P} \subset \mathcal{E}$, the map $\delta_{T(\mathcal{E})}$ being restricted to $S(\mathcal{P}) \otimes \Lambda(\mathcal{P})$ via the embedding $s y m_{\mathcal{P}} \otimes i d_{\mathcal{P}}$ gives a derivation of the algebra $S(\mathcal{P}) \otimes \Lambda(\mathcal{P})$; we denote it by $\delta_{\mathcal{P}}$.

On the algebra $S(\mathcal{P}) \otimes \Lambda(\mathcal{P})$, there is another derivation, $\delta_{\mathcal{P}}^{*}$, of degree -1 generated by the map $1 \otimes \Lambda^{1}(\mathcal{P}) \rightarrow S^{1}(\mathcal{P}) \otimes 1,1 \otimes v \rightarrow v \otimes 1, v \in \mathcal{P}$. It is easy to check that $\left(\delta_{\mathcal{P}}^{*}\right)^{2}=0$ and $\left[\delta_{\mathcal{P}}, \delta_{\mathcal{P}}^{*}\right]=\delta_{\mathcal{P}} \delta_{\mathcal{P}}^{*}+\delta_{\mathcal{P}}^{*} \delta_{\mathcal{P}}=\operatorname{deg}$, where deg is the derivation assigning to an element $x \in S^{p}(\mathcal{P}) \otimes \Lambda^{q}(\mathcal{P})$ the element $(p+q) x$. 
Let $\mathcal{E}$ be presented as a direct sum of $\mathcal{C}_{M}^{\infty}[[t]]$-submodules, $\mathcal{E}=\mathcal{P} \oplus \mathcal{Q}$. It is obvious that the derivations $\delta_{\mathcal{P}}, \delta_{\mathcal{P}}^{*}, \delta_{\mathcal{Q}}, \delta_{\mathcal{Q}}^{*}$ induce derivations on the algebra $\mathbf{S}(\mathcal{P}, \mathcal{Q})=(S(\mathcal{P}) \otimes$ $S(\mathcal{Q})) \otimes(\Lambda(\mathcal{P}) \otimes \Lambda(\mathcal{Q}))$ that we will identify in the natural way with the algebra $(S(\mathcal{P}) \otimes$ $S(\mathcal{Q})) \otimes \Lambda(\mathcal{E})$. We put $\delta_{\mathcal{P}, \mathcal{Q}}=\delta_{\mathcal{P}}+\delta_{\mathcal{Q}}$ and $\delta_{\mathcal{P}, \mathcal{Q}}^{*}=\delta_{\mathcal{P}}^{*}+\delta_{\mathcal{Q}}^{*}$.

Let us define the operator $\delta_{\mathcal{P}, \mathcal{Q}}^{-1}$ on $\mathbf{S}(\mathcal{P}, \mathcal{Q})$ in the following way. We put $\delta_{\mathcal{P}, \mathcal{Q}}^{-1}(x)=0$ for $x \in \mathcal{C}_{M}^{\infty}[[t]]$ and $\delta_{\mathcal{P}, \mathcal{Q}}^{-1}(x)=\left(1 /(p+r+q) \delta_{\mathcal{P}, \mathcal{Q}}^{*}(x)\right.$ for $x \in\left(S^{p}(\mathcal{P}) \otimes S^{r}(\mathcal{Q})\right) \otimes \Lambda^{q}(\mathcal{E})$, $p+r+q>0$. There is the obvious relation

$$
\delta_{\mathcal{P}, \mathcal{Q}} \delta_{\mathcal{P}, \mathcal{Q}}^{-1}+\delta_{\mathcal{P}, \mathcal{Q}}^{-1} \delta_{\mathcal{P}, \mathcal{Q}}=\text { projection on } \mathbf{S}^{+}(\mathcal{P}, \mathcal{Q}) \text { along } \mathcal{C}_{M}^{\infty}[[t]]
$$

where $\mathbf{S}^{+}(\mathcal{P}, \mathcal{Q})$ is the closure of $\oplus_{p+r+q>0}\left(S^{p}(\mathcal{P}) \otimes S^{r}(\mathcal{Q})\right) \otimes \Lambda^{q}(\mathcal{E})$.

One has the embedding

$$
\operatorname{sym}_{\mathcal{P}} \otimes \operatorname{sym}_{\mathcal{Q}} \otimes i d:(S(\mathcal{P}) \otimes S(\mathcal{Q})) \otimes \Lambda(\mathcal{E}) \rightarrow T(\mathcal{E}) \otimes \Lambda(\mathcal{E}) .
$$

It is clear that $\delta_{\mathcal{P}, \mathcal{Q}}$ coincides with the restriction of $\delta_{T(\mathcal{E})}$ to $\mathbf{S}(\mathcal{P}, \mathcal{Q})$ via this embedding.

\subsection{The Fedosov algebra}

Let $\varphi: \mathcal{E} \otimes \mathcal{E} \rightarrow \mathcal{C}_{M}^{\infty}[[t]]$ be a $\mathcal{C}_{M}^{\infty}[[t]]$-linear skew-symmetric nondegenerate form and $I$ the closed ideal in $T(\mathcal{E})$ generated by relations

$$
x \otimes y-y \otimes x=t \varphi(x, y) .
$$

We call $\mathcal{W}(\mathcal{E})=T(\mathcal{E}) / I$ the Weyl algebra and $\mathbf{W}=\mathbf{W}(\mathcal{E})=\mathcal{W} \otimes \Lambda(\mathcal{E})$ the Fedosov algebra over $\mathcal{E}$. The derivation $\delta_{T(\mathcal{E})}$ on $T(\mathcal{E}) \otimes \Lambda(\mathcal{E})$ induces a derivation of $\mathbf{W}$. Indeed, $\delta_{T(\mathcal{E})}$ applied to the both sides of (9.4) gives zero. We denote this derivation by $\delta$.

Let $\mathcal{E}=\mathcal{P} \oplus \mathcal{Q}$ be a decomposition into $\mathcal{C}_{M}^{\infty}[[t]]$-modules.

Define the Wick map, $\mathbf{w}_{\mathcal{P}, \mathcal{Q}}$, as the composition $\mathbf{S}(\mathcal{P}, \mathcal{Q}) \rightarrow T(\mathcal{E}) \otimes \Lambda(\mathcal{E}) \rightarrow \mathbf{W}$, where the first map is (9.3) and the second one is the projection. By the PBW theorem $\mathbf{w}_{\mathcal{P}, \mathcal{Q}}$ is an isomorphism of $\mathcal{C}_{M}^{\infty}[[t]]$-modules.

Due to the isomorphism $\mathbf{w}_{\mathcal{P}, \mathcal{Q}}$, the operators $\delta_{\mathcal{P}, \mathcal{Q}}$ and $\delta_{\mathcal{P}, \mathcal{Q}}^{-1}$ are carried over from $\mathbf{S}(\mathcal{P}, \mathcal{Q})$ to $\mathbf{W}$. We retain for them the same notation. Note that while $\delta_{\mathcal{P}, \mathcal{Q}}$ does not depend on the decomposition of $\mathcal{E}$ and coincides with the derivation $\delta$ induced from $T(\mathcal{E}) \otimes \Lambda(\mathcal{E})$, the operator $\delta_{\mathcal{P}, \mathcal{Q}}^{-1}$ is not a derivation and does depend on the decomposition. In particular, one can suppose that the decomposition is trivial, $\mathcal{E}=\mathcal{E} \oplus 0$. In this case we denote $\delta_{\mathcal{E}}^{-1}=\delta_{\mathcal{E}, 0}^{-1}$. 
Proposition 9.1. One has

$$
H(\mathbf{W}, \delta)=\mathcal{C}_{M}^{\infty}[[t]] .
$$

Moreover, if $x \in \mathcal{W}(\mathcal{E}) \otimes \Lambda^{k>0}(\mathcal{E})$ then $y=\delta_{\mathcal{P}, \mathcal{Q}}^{-1} x$ is such that $\delta y=x$ for any decomposition $\mathcal{E}=\mathcal{P} \oplus \mathcal{Q}$.

Proof. Follows from (9.2).

\subsection{Lie subalgebras in $\mathcal{W}$}

Let $\mathcal{E}=\mathcal{P} \oplus \mathcal{Q}$ be a decomposition. We say that $x \in \mathbf{W}$ has $\mathbf{w}_{\mathcal{P}, \mathcal{Q}}$-degree $(p, q)$ if $\mathbf{w}_{\mathcal{P}, \mathcal{Q}}^{-1}(x) \in\left(S^{p}\left(\mathcal{P} \otimes S^{q}(\mathcal{Q})\right) \otimes \Lambda(\mathcal{E})\right.$. We say that $x \in \mathbf{W}$ has $\mathbf{w}_{\mathcal{P}, \mathcal{Q}}$-degree $k$ if $\mathbf{w}_{\mathcal{P}, \mathcal{Q}}^{-1}(x) \in$ $\oplus_{p+q=k}\left(S^{p}(\mathcal{P}) \otimes S^{q}(\mathcal{Q})\right) \otimes \Lambda(\mathcal{E})$. We define the $\mathbf{w}_{\mathcal{E}}$-degree as the $\mathbf{w}_{\mathcal{E}, 0}$-degree for the trivial decomposition $\mathcal{E}=\mathcal{E} \oplus 0$.

Let $\mathfrak{g}$ be a sheaf of Lie algebras acting on $\mathcal{E}$. We call a $\mathcal{C}_{M}^{\infty}[[t]]$-linear map $\lambda: \mathfrak{g} \rightarrow \mathcal{W}$ a realization of $\mathfrak{g}$, if it is a Lie algebra morphism $(\mathcal{W}$ is considered as a Lie algebra with respect to the commutator $\left.\frac{1}{t}[\cdot, \cdot]\right)$ and for any $x \in \mathfrak{g}$ and $v \in \mathcal{E}$ one has $x(v)=\frac{1}{t}[\lambda(x), v]$. It is easy to check that any two realizations differ by a Lie algebra morphism of $\mathfrak{g}$ to the center of $\mathcal{W}$, so if $\mathfrak{g}$ is a sheaf of semisimple Lie algebras, there is not more than one realization of $\mathfrak{g}$.

Denote by $\mathfrak{s p}(\mathcal{E})$ the sheaf of symplectic Lie algebras with respect to $\varphi$. Since $\mathfrak{s p}(\mathcal{E})$ is semisimple, there is a unique realization $\rho_{\mathcal{E}}: \mathfrak{s p}(\mathcal{E}) \rightarrow \mathcal{W}$. The image of this realization consists of elements having $\mathbf{w}_{\mathcal{E}}$-degree two.

Let $\mathcal{E}=\mathcal{P} \oplus \mathcal{Q}$ be a decomposition into Lagrangian subsheaves. Denote by $\mathfrak{s p}(\mathcal{P}, \mathcal{E})$ the subsheaf of $\mathfrak{s p}(\mathcal{E})$ preserving $\mathcal{P}$. It is easy to check that $\mathfrak{s p}(\mathcal{P}, \mathcal{E})$ can be realized as the subset of elements of $\mathcal{W}$ having $\mathbf{w}_{\mathcal{P}, \mathcal{Q}}$-degree $(1,1)$ and $(2,0)$. Denote this realization by $\rho_{\mathcal{P}, \mathcal{E}}: \mathfrak{s p}(\mathcal{P}, \mathcal{E}) \rightarrow \mathcal{W}$. On the other hand, $\mathfrak{s p}(\mathcal{P}, \mathcal{E})$ is realized in $\mathcal{W}$ by $\rho_{\mathcal{E}}$.

Lemma 9.2. Let $b \in \mathfrak{s p}(\mathcal{P}, \mathcal{E})$. Then

$$
\rho_{\mathcal{E}}(b)=\rho_{\mathcal{P}, \mathcal{E}}(b)+\frac{t}{2} \operatorname{trace}(\bar{b}),
$$

where $\bar{b}$ is $b$ restricted to $\mathcal{P}$.

Proof. Direct computation using the fact that $\rho_{\mathcal{P}, \mathcal{E}}(\bar{b})$ is $(1,1) \mathbf{w}_{\mathcal{P}, \mathcal{Q}}$-degree component of $\rho_{\mathcal{P}, \mathcal{E}}(b)$ in any decomposition $\mathcal{E}=\mathcal{P} \oplus \mathcal{Q}$. 


\subsection{Filtrations on $\mathcal{W}$}

We define two decreasing filtrations on $\mathcal{W}$ numbered by nonnegative integers.

The $T$-filtration $F_{\bullet}^{T} \mathcal{W}$ is defined as follows. We ascribe to the elements of $\mathcal{E}$ degree 1 and to $t$ degree 2. Then $F_{n}^{T} \mathcal{W}$ consists of elements of $\mathcal{W}$ having the leading term of total degree $\geq n$.

The $\mathcal{P}$-filtration, $F_{\bullet}^{\mathcal{P}} \mathcal{W}$, is firstly defined on $S(\mathcal{P}) \otimes S(\mathcal{Q})$ by the subsets $F_{n}^{\mathcal{P}}=$ $\oplus_{i \geq n} S^{i}(\mathcal{P}) \otimes S(\mathcal{Q}), n=0,1, \ldots$, and carried over to $\mathcal{W}$ via the Wick isomorphism.

We extend those filtrations to $\mathbf{W}$ in the natural way standing, for example, $F_{n}^{T} \mathbf{W}=$ $F_{n}^{T} \mathcal{W} \otimes \Lambda(\mathcal{E})$. We will use the following mnemonic notation. To point out, for example, that a section $x \in \mathbf{W}$ belongs to $F_{n}^{T} \mathbf{W}$ we write $F^{T}(x) \geq n$.

In the following we denote $\mathbf{S}(\mathcal{P})=S(\mathcal{P}) \otimes \Lambda(\mathcal{E})$ embedded in $\mathbf{S}(\mathcal{P}, \mathcal{Q})$ as $(S(\mathcal{P}) \otimes 1) \otimes$ $\Lambda(\mathcal{E})$.

Proposition 9.3. Let $\mathcal{E}=\mathcal{P} \oplus \mathcal{Q}$ be a decomposition of $\mathcal{E}$ into Lagrangian subsheaves. Then

a) The Wick map $\mathbf{w}_{\mathcal{P}, \mathcal{Q}}: \mathbf{S}(\mathcal{P}, \mathcal{Q}) \rightarrow \mathbf{W}$ has the following property: for a $\in \mathbf{S}(\mathcal{P})$ and arbitrary $c \in \mathbf{S}(\mathcal{P}, \mathcal{Q})$ one has ac $=\mathbf{w}(a) \mathbf{w}(c)$.

The filtrations on $\mathbf{W}$ have the properties:

b) for $x, y \in \mathbf{W}$, if $F^{\mathcal{P}}(x) \geq k$, then $F^{\mathcal{P}}(x y) \geq k$;

c) $F^{\mathcal{P}}\left(\delta_{\mathcal{P}, \mathcal{Q}}^{-1} x\right) \geq F^{\mathcal{P}}(x)$;

d) $F^{T}\left(\delta_{\mathcal{P}, \mathcal{Q}}^{-1} x\right)>F^{T}(x)$.

Proof. Clear.

\subsection{Fedosov's construction in the Wick case}

Let $\left(M, \omega_{t}, \mathcal{P}_{t}\right)$ be a formal polarized symplectic manifold. We will write for shortness $\omega=\omega_{t}, \mathcal{P}=\mathcal{P}_{t}$. Let us denote $\mathcal{T}=\mathcal{T}_{M}^{\mathbb{C}}[[t]]$. It is easy to prove that there exists on $M$ a Lagrangian subbundle $\mathcal{Q} \subset \mathcal{T}$ such that $\mathcal{T}=\mathcal{P} \oplus \mathcal{Q}$.

In the following we set $\mathcal{E}=\mathcal{T}^{*}$ and consider the Fedosov algebra $\mathbf{W}=\mathbf{W}(\mathcal{E})$ with respect to $\varphi$ being the Poisson bracket inverse to $\omega$. The decomposition $\mathcal{E}=\mathcal{P}^{\perp} \oplus \mathcal{Q}^{\perp}$ is Lagrangian with respect to this $\varphi$.

Let $\nabla$ be a $\mathcal{P}$-symplectic connection on $M$ (see Section 5). Then the induced connection $\nabla: \mathcal{E} \rightarrow \mathcal{E} \otimes \Lambda^{1} \mathcal{E}$ on $\mathcal{E}$ preserves $\mathcal{P}^{\perp}$, i.e. $\nabla\left(\mathcal{P}^{\perp}\right) \subset \mathcal{P}^{\perp} \otimes \Lambda^{1}(\mathcal{E})$, and is flat on $\mathcal{P}^{\perp}$ along $\mathcal{P}$, i.e. for any $X, Y \in \mathcal{P}$ one has $\left(\nabla_{X} \nabla_{Y}-\nabla_{Y} \nabla_{X}-\nabla_{[X, Y]}\right)\left(\mathcal{P}^{\perp}\right)=0$. 
We will identify $\mathcal{P}$ with $\mathcal{P}^{\perp}$ and $\mathcal{Q}$ with $\mathcal{Q}^{\perp}$ by the isomorphism $x \mapsto \omega(x, \cdot)$ between $\mathcal{T}$ and $\mathcal{E}$. So, we will allow us to write $\mathcal{E}=\mathcal{P} \oplus \mathcal{Q}$.

The connection $\nabla$ gives a derivation of the Fedosov algebra $\mathbf{W}$, which is an extension of the de Rham differential on functions. Analogously, $\nabla$ gives such derivations of the algebras $T(\mathcal{E}) \otimes \Lambda(\mathcal{E}), S(\mathcal{E}) \otimes \Lambda(\mathcal{E})$, and $(S(\mathcal{P}) \otimes S(\mathcal{Q})) \otimes \Lambda(\mathcal{E})$. These derivations commute with the maps $(9.3)$ and $\mathbf{w}_{\mathcal{P}, \mathcal{Q}}$.

For convenience, we will mark the elements of the Fedosov algebra lying in $\mathcal{E} \otimes 1$ by letters with hat over them $(\hat{x})$, while by $d x$ we will denote the copy of $\hat{x}$ lying in $1 \otimes \Lambda^{1} \mathcal{E}$.

Let $\omega=\omega_{i j} d x_{i} \wedge d x_{j}$ in some local coordinates. It is easy to check that for $\widetilde{\delta}=$ $\omega_{i j} \hat{x}_{i} \otimes d x_{j}$ one has

$$
\begin{aligned}
\delta & =\frac{1}{t} \operatorname{ad}(\widetilde{\delta}), \\
\widetilde{\delta}^{2} & =t \omega .
\end{aligned}
$$

Since the torsion of $\nabla$ is equal to zero,

$$
\nabla(\widetilde{\delta})=0
$$

Since $\nabla^{2}$ is a $\mathcal{C}_{M}^{\infty}[[t]]$-linear derivation of degree 0 preserving $\mathcal{P}$, there is an element $R \in$ $\rho_{\mathcal{P}, \mathcal{E}}(\mathfrak{s p}(\mathcal{P}, \mathcal{E})) \otimes \Lambda^{2}(\mathcal{E})$ such that $\nabla^{2}=\frac{1}{t} \operatorname{ad}(R)$. In particular, one has to be

$$
F^{\mathcal{P}}(R) \geq 1
$$

According to Fedosov, [Fe], we also define $R^{F} \in \rho_{\mathcal{E}}(\mathfrak{s p}(\mathcal{P}, \mathcal{E})) \otimes \Lambda^{2}(\mathcal{E})$ satisfying $\nabla^{2}=$ $\frac{1}{t} \operatorname{ad}\left(R^{F}\right)$. It follows from (9.6)

$$
\delta(R)=\delta\left(R^{F}\right)=0
$$

Following to Fedosov, we will consider connections on $\mathbf{W}$ of the form

$$
D=\nabla+\frac{1}{t} \operatorname{ad}(\gamma), \quad \gamma \in \mathcal{W} \otimes \Lambda^{1}(\mathcal{E})
$$

We define the Wick curvature of $D$ as

$$
\Omega_{D}=R+\nabla(\gamma)+\frac{1}{t} \gamma^{2}
$$

According to Fedosov, we also define the Weyl (or Fedosov) curvature of $D$ as

$$
\Omega_{D}^{F}=R^{F}+\nabla(\gamma)+\frac{1}{t} \gamma^{2}
$$


Since by Lemma $9.2 R^{F}=R+\frac{t}{2} \operatorname{tr}\left(\left.\nabla^{2}\right|_{\mathcal{P}}\right)$, we have

$$
\Omega_{D}^{F}=\Omega_{D}+\frac{t}{2} \operatorname{tr}\left(\left.\nabla^{2}\right|_{\mathcal{P}}\right)
$$

One checks

$$
D^{2}=\frac{1}{t} \operatorname{ad}\left(\Omega_{D}\right)=\frac{1}{t} \operatorname{ad}\left(\Omega_{D}^{F}\right)
$$

Let us take $\gamma$ in the form

$$
\gamma=\widetilde{\delta}+r, \quad r \in \mathcal{W} \otimes \Lambda^{1}(\mathcal{E}), \quad F^{T}(r) \geq 3 .
$$

Then the connection $D$ has the form

$$
\nabla+\delta+\frac{1}{t} \operatorname{ad}(r)
$$

Using (9.5) and (9.6), we obtain that its Wick curvature is

$$
\Omega_{D}=R+\nabla(\widetilde{\delta}+r)+\frac{1}{t}(\widetilde{\delta}+r)^{2}=\omega+\delta r+R+\nabla r+\frac{1}{t} r^{2} .
$$

Proposition 9.4. There exists an element $r \in \mathcal{W}(\mathcal{E}) \otimes \Lambda^{1}(\mathcal{E})$ such that

a) $F^{T}(r) \geq 3$;

b) $F^{\mathcal{P}}(r) \geq 1$;

c) the connection $D=\nabla+\delta+\frac{1}{t} \operatorname{ad}(r)$ is flat, i.e. $D^{2}=0$;

d) for its Wick curvature one has

$$
\Omega_{D}=\omega
$$

e) its Weyl curvature $\Omega_{D}^{F}$ belong to $\Gamma\left(M, d \mathcal{P}^{\perp}\right)$ and there is the formula

$$
\Omega_{D}^{F}=\omega+\frac{t}{2} \operatorname{tr}\left(\left.\nabla^{2}\right|_{\mathcal{P}}\right)
$$

Proof. First of all, we apply the Fedosov method, ( $[\mathrm{Fe}]$, Theorem 5.2.2), to find $r$ satisfying d). According to (9.15), $r$ must obey the equation

$$
\delta r=-\left(R+\nabla r+\frac{1}{t} r^{2}\right)
$$

Let us look for $r$ being the limit of the sequence, $r=\lim r_{k}$, where $r_{k} \in \mathcal{W}(\mathcal{E}) \otimes \Lambda^{1}(\mathcal{E})$, $k=3,4, \ldots$, and $F^{T}\left(r_{k}-r_{k-1}\right) \geq k$. As in Lemma 5.2.3 of [Fe], using Proposition 9.3 d) and the fact that $F^{T}(R) \geq 2$, such $r_{k}$ can be calculated recursively:

$$
\begin{aligned}
r_{3} & =-\delta_{\mathcal{P}, \mathcal{Q}}^{-1}(R) \\
r_{k+3} & =-\left(r_{3}+\delta_{\mathcal{P}, \mathcal{Q}}^{-1}\left(\nabla r_{k+2}+\frac{1}{t} r_{k+2}^{2}\right)\right) .
\end{aligned}
$$


So, a) and d) are proven.

Let us prove that $F^{\mathcal{P}}\left(r_{k}\right) \geq 1$ for all $k$. The inequality $F^{\mathcal{P}}\left(r_{3}\right) \geq 1$ follows from the fact that $F^{\mathcal{P}}(R) \geq 1$ and from Proposition (9.3) c). Suppose that $F^{\mathcal{P}}\left(r_{i}\right) \geq 1$ for $i<k+3$, $k>0$. Then $F^{\mathcal{P}}\left(\nabla r_{k+2}\right) \geq 1$ because $\nabla$ preserves $\mathcal{P}$. On the other hand, $F^{\mathcal{P}}\left(r_{k+2}^{2}\right) \geq 1$ because of Proposition (9.3) b), therefore, as follows from (9.18), $F^{\mathcal{P}}\left(r_{k+3}\right) \geq 1$ as well. So, we have that $r$ being the limit of the convergent sequence $r_{k}$ satisfies the conditions a), b), and d) of the proposition.

c) obviously follows from d) and (9.12).

e) follows immediately from d), (9.11), and Lemma 5.3.

Proposition 9.5. Let $\widetilde{\nabla}$ be another $\mathcal{P}$-symplectic connection on $M$. Let $\widetilde{r} \in \mathcal{W}(\mathcal{E}) \otimes$ $\Lambda^{1}(\mathcal{E})$, satisfy the conclusions of Proposition 9.4, in particular, the connection $\widetilde{D}=\widetilde{\nabla}+$ $\delta+\frac{1}{t} \operatorname{ad}(\widetilde{r})$ is flat. Then, there exists an element $B \in \mathcal{W}(\mathcal{E})$ such that
a) $F^{T}(B) \geq 3$;
b) $F^{\mathcal{P}}(B) \geq 1$
c) $e^{\frac{1}{t} \operatorname{ad} B} D=\widetilde{D}$.

Proof. Note that $\nabla-\widetilde{\nabla}$ can be presented as $\frac{1}{t} \operatorname{ad} R_{0}$, where $R_{0} \in \rho_{\mathcal{P}, \mathcal{E}}(\mathfrak{s p}(\mathcal{P}, \mathcal{E}))$. Therefore, $F^{T}\left(R_{0}\right) \geq 2$ and $F^{\mathcal{P}}\left(R_{0}\right) \geq 1$. Let us put $R_{1}=r-\widetilde{r}$. Then, $F^{T}\left(R_{1}\right) \geq 3$ and $F^{\mathcal{P}}\left(R_{1}\right) \geq 1$. We have

$$
\widetilde{D}=D-\frac{1}{t} \operatorname{ad}\left(R_{0}+R_{1}\right)
$$

Since $\Omega_{D}=\Omega_{\widetilde{D}}=\omega$, using (9.6) we obtain

$$
\delta\left(R_{0}\right)=0
$$

It follows that the element $B_{0}=\delta_{\mathcal{P}, \mathcal{Q}}^{-1}\left(R_{0}\right)$ is such that $\delta\left(B_{0}\right)=R_{0}$ and $F^{T}\left(B_{0}\right) \geq 3$, $F^{\mathcal{P}}\left(B_{0}\right) \geq 1$.

Replacing $D$ by $e^{\frac{1}{t} \text { ad } B_{0}} D$ we obtain

$$
\widetilde{D}=D-\frac{1}{t} \operatorname{ad}\left(R_{0}^{\prime}+R_{1}^{\prime}\right)
$$

where $F^{T}\left(R_{0}^{\prime}\right) \geq 3, F^{\mathcal{P}}\left(R_{0}^{\prime}\right) \geq 1$ and $F^{T}\left(R_{1}^{\prime}\right) \geq 4, F^{\mathcal{P}}\left(R_{1}^{\prime}\right) \geq 1$.

Proceeding by induction on $F^{T}$-filtration, we obtain a sequence $B_{i} \in \mathcal{W}(\mathcal{E})$ with increasing $F^{T}$-filtration and such that $\prod_{i=0}^{\infty} e^{\frac{1}{t} \operatorname{ad} B_{i}}(D)=\widetilde{D}$.

Since elements $e^{\frac{1}{t} \operatorname{ad} B^{\prime}}, B^{\prime} \in \mathcal{W}(\mathcal{E}), F^{T}(B) \geq 3$, form a pro-unipotent Lie group, there exists an element $B \in \mathcal{W}(\mathcal{E}), F^{T}(B) \geq 3, F^{\mathcal{P}}(B) \geq 1$, such that $\prod_{i=0}^{\infty} e^{\frac{1}{t} \operatorname{ad} B_{i}}=e^{\frac{1}{t} \operatorname{ad} B}$. 
Let $D$ be a connection satisfying Proposition 9.4. Denote by $\mathcal{W}_{D}$ the subsheaf of $\mathcal{W}$ consisting of flat sections $a$, i.e. such that $D a=0$. Since $D$ is a derivation of $\mathbf{W}$, it is clear that $\mathcal{W}_{D}$ is a sheaf of subalgebras. Let $\sigma=i d-\left(\delta \delta_{\mathcal{P}, \mathcal{Q}}^{-1}+\delta_{\mathcal{P}, \mathcal{Q}}^{-1} \delta\right)$. Then, as follows from (9.2), $\sigma: \mathcal{W} \rightarrow \mathcal{C}_{M}^{\infty}[[t]]$ is a projection, where $\mathcal{C}_{M}^{\infty}[[t]]$ is considered as the center of the algebra $\mathcal{W}$.

Proposition 9.6. a) The map $\sigma: \mathcal{W}_{D} \rightarrow \mathcal{C}_{M}^{\infty}[[t]]$ is a bijection.

b) The inverse map $\left.\left.\eta: \mathcal{C}_{M}^{\infty}[t]\right]\right] \rightarrow \mathcal{W}_{D}$ has the form $\eta(f)=f+\hat{f}$, there $F^{T}(\hat{f})>F^{T}(f)$.

c) If $d f \in \mathcal{P}$, then $F^{\mathcal{P}}(\hat{f}) \geq 1$.

d) If $d f \in \mathcal{P}$, then $\sigma(\eta(f) \eta(g))=$ fg for any $g \in \mathcal{C}_{M}^{\infty}[[t]]$.

Proof. Again, we apply the Fedosov iteration procedure. According to [F], Theorem 5.2.4, we look for $\eta(f)$ as a limit, $\eta(f)=\lim a_{k}$, there $a_{k} \in \mathcal{W}$ can be calculated recursively:

$$
\begin{aligned}
a_{0} & =f \\
a_{k+1} & =a_{0}+\delta_{\mathcal{P}, \mathcal{Q}}^{-1}\left(\nabla a_{k}+\frac{1}{t} \operatorname{ad} r\left(a_{k}\right)\right) .
\end{aligned}
$$

Put $\hat{f}=\eta(f)-a_{0}$. As in $[\mathrm{Fe}]$, Theorem 5.2.4, one proves that such $\eta(f)$ and $\hat{f}$ satisfy a) and b). Now observe that $a_{1}-a_{0}=\delta_{\mathcal{P}, \mathcal{Q}}^{-1}(1 \otimes d f)$ and if $d f \in \mathcal{P}$, then $F^{\mathcal{P}}\left(a_{1}-a_{0}\right) \geq 1$. By induction, we conclude that $F^{\mathcal{P}}\left(a_{k}-a_{0}\right) \geq 1$ for all $k \geq 1$. So $F^{\mathcal{P}}\left(a-a_{0}\right) \geq 1$ as well, which proves c).

Let us prove d). We have $\eta(f) \eta(g)=f \eta(g)+\hat{f} \eta(g)$. Since by c) $F^{\mathcal{P}}(\hat{f}) \geq 1$, $F^{\mathcal{P}}(\hat{f} \eta(g)) \geq 1$ as well. It follows that $\sigma(\hat{f} \eta(g))=0$ and $\sigma(\eta(f) \eta(g))=\sigma(f \eta(g))=f g$, because $\sigma$ is a $\mathcal{C}_{M}^{\infty}[[t]]$-linear map and $\sigma(\eta(g))=g$.

\subsection{Existence of PSP's}

Let $(M, \omega, \mathcal{P})$ be a polarized symplectic manifold. Recall that in Subsection 8.3 we have assigned to any PSP $\left(\mu_{t}, \mathcal{O}_{t}\right)$ on $(M, \omega, \mathcal{P})$ an element $\tau\left(\mu_{t}, \mathcal{O}_{t}\right) \in \mathcal{Y}(M, \omega, \mathcal{P})$, which is a pair $\left(\omega_{t}, \mathcal{P}_{t}\right)$ being a deformation of the pair $(\omega, \mathcal{P})$. The form $\omega_{t}$ represents the extension class $c l_{E x t}\left(\mu_{t}, \mathcal{O}_{t}\right)$.

We show now that any element of $\mathcal{Y}(M, \omega, \mathcal{P})$ corresponds to a PSP.

Proposition 9.7. a) For any pair $\left(\omega_{t}, \mathcal{P}_{t}\right) \in \mathcal{Y}(M, \omega, \mathcal{P})$, there exists a PSP, $\left(\mu_{t}, \mathcal{O}_{t}\right)$, such that

$$
\tau\left(\mu_{t}, \mathcal{O}_{t}\right)=\left(\omega_{t}, \mathcal{P}_{t}\right)
$$


b) The Fedosov class of the corresponding star-product $\left(\mathcal{C}_{M}^{\infty}[[t]], \mu_{t}\right)$ is represented by the form of $\omega+t \Gamma\left(M, d\left(\mathcal{P}_{t}^{\perp}\right)\right)$ equal to

$$
\theta_{t}=\omega_{t}+\frac{t}{2} \operatorname{tr}\left(\left.\nabla^{2}\right|_{\mathcal{P}_{t}}\right)
$$

where $\nabla$ is a $\mathcal{P}_{t}$-symplectic connection on the formal symplectic manifold $\left(M, \omega_{t}, \mathcal{P}_{t}\right)$.

Proof. Let $\mathbf{W}$ be the Fedosov algebra on $M$ corresponding to the symplectic form $\omega_{t}$. Let $\nabla$ be a $\mathcal{P}_{t}$-symplectic connection on $M$ corresponding to $\omega_{t}$ and $D$ the flat connection on W constructed in Proposition 9.4 c). Let $\mathcal{W}_{D}$ be the sheaf of flat sections of $\mathcal{W}$. Define a star-product $\left(\mathcal{C}_{M}^{\infty}[[t]], \mu_{t}\right)$ on $M$ carrying over the multiplication from $\mathcal{W}_{D}$ to $\mathcal{C}_{M}^{\infty}[[t]]$ via the map $\sigma$ from Proposition 9.6. Point d) of that proposition shows that, in fact, this starproduct present the PSP $\left(\mathcal{C}_{M}^{\infty}[[t]], \mu_{t}, \mathcal{O}_{\mathcal{P}_{t}}\right)$. We are going to prove that this star-product is as required. In the following we identify $\mathcal{W}_{D}$ with the corresponding PSP via $\sigma$.

Let us prove (9.20). Let $\left(U_{\alpha}\right)$ be an open covering of $M$ such that on each $U_{\alpha}$ there exist formal Darboux coordinates $x_{\alpha i}, y_{\alpha i}, x_{\alpha i} \in \mathcal{O}_{\mathcal{P}_{t}}$ with respect to $\omega_{t}$.

Denote by $\nabla_{\alpha}$ the standard flat $\mathcal{P}_{t}$-symplectic connections over $U_{\alpha}$ such that the forms $d x_{\alpha i}, d y_{\alpha i}$ are flat sections in $\mathcal{P}_{t}^{\perp}$. Then, the connections $D_{\alpha}=\nabla_{\alpha}+\delta$ satisfy on $U_{\alpha}$ Proposition 9.4 with $r=0$. Let $\mathcal{W}_{D_{\alpha}}$ be the star-product on $U_{\alpha}$ constructed in Proposition 9.6 via flat sections of $D_{\alpha}$. It is easy to see that $\mathcal{W}_{D_{\alpha}}$ coincides with the Moyal-Wick PSP with respect to the Darboux coordinates $x_{\alpha i}, y_{\alpha i}$ (see Example 7.15), so $x_{\alpha i}, y_{\alpha i}$ are also Darboux coordinates for the bracket $\frac{1}{t}[\cdot, \cdot]$ in $\mathcal{W}_{D_{\alpha}}$.

Since $D$ and $D_{\alpha}$ have the same Wick curvature $\omega_{t}$, there exist, by Proposition 9.5, elements $B_{\alpha} \in \mathcal{W}$ such that $e^{\frac{1}{t} \operatorname{ad} B_{\alpha}} D_{\alpha}=D$. It is clear that $e^{\frac{1}{t} \operatorname{ad} B_{\alpha}}$ acting on $\mathcal{W}$ takes $\mathcal{W}_{D_{\alpha}}$ to $\mathcal{W}_{D}$, and point b) of that Proposition implies that it is identical on $\mathcal{O}_{\mathcal{P}_{t}}$.

Let $\Psi_{\alpha, \beta}=e^{-\frac{1}{t} \mathrm{ad} B_{\alpha}} e^{\frac{1}{t} \mathrm{ad} B_{\beta}}$. These may be considered as isomorphisms over $U_{\alpha} \cap U_{\beta}$ gluing star-products $\mathcal{W}_{D_{\alpha}}$ to a global star-product on $M$ that is obviously isomorphic to $\mathcal{W}_{D}$. We see, that functions $x_{\alpha i}, y_{\alpha i}$ form local Darboux coordinates corresponding to that star-product. So, the characteristic 2-form $\operatorname{cl}_{P S P}\left(\mathcal{W}_{D}\right)$ (see Subsection 8.3) is locally represented as $d y_{\alpha i} \wedge d x_{\alpha i}$. On the other hand, this form is equal to $\omega_{t}$, since from very beginning the functions $x_{\alpha i}, y_{\alpha i}$ have been chosen as Darboux coordinates for it. Hence, $\tau\left(\mathcal{W}_{D}\right)=\left(\omega_{t}, \mathcal{P}_{t}\right)$.

b) Follows from Proposition 9.4 e) and Lemma 5.3 . 


\section{The main theorem and corollaries}

Let $(M, \omega, \mathcal{P})$ be a polarized symplectic manifold. Denote by $\mathcal{Y}$ the set of pairs $\left(\omega_{t}, \mathcal{P}_{t}\right)$, where $\omega_{t}=\omega+t \omega_{1}+\cdots$ is a deformed symplectic form and $\mathcal{P}_{t}, \mathcal{P}_{0}=\mathcal{P}$, its polarization. Let $\operatorname{Aut}(M)$ be the group of formal automorphisms of $M$.

Theorem 10.1. a) The equivalence classes of $P D Q$ 's on $(M, \omega, \mathcal{P})$ are in one-to-one correspondence with the orbits in $\mathcal{Y}$ under the Aut $(M)$-action.

b) Let the pair $\left(\omega_{t}, \mathcal{P}_{t}\right)$ be a point on the orbit corresponding to a $P D Q\left(\mathbb{A}_{t}, \mathbb{O}_{t}\right)$. Then, $\left(\mathbb{A}_{t}, \mathbb{O}_{t}\right)$ is isomorphic to a PSP, $\left(\mathcal{C}_{M}^{\infty}[[t]], \mu_{t}, \mathcal{O}_{t}\right)$, where $\mathcal{O}_{t}$ consists of functions constant along $\mathcal{P}_{t}$ and the multiplication $\mu_{t}$ satisfies the condition

$$
\mu_{t}(f, g)=f g \quad \text { for } f \in \mathcal{O}_{t}, g \in \mathcal{C}_{M}^{\infty}[[t]]
$$

c) The form $\omega_{t}$ represents the extension class $c l_{E x t}\left(\mathbb{A}_{t}, \mathbb{O}_{t}\right) \in H^{1}\left(M, \Omega_{\mathcal{O}_{t}}^{1 c l}\right)$, associated with $\left(\mathbb{A}_{t}, \mathbb{O}_{t}\right)$.

d) Under the hypothesis of $b$ ), the Fedosov class of the deformation quantization $\mathbb{A}_{t}$ can be represented by a form $\theta_{t}$ that is a deformation of $\omega$ and polarized by $\mathcal{P}_{t}$. It is defined by the formula

$$
\theta_{t}=\omega_{t}+\frac{t}{2} \operatorname{tr}\left(\left.\nabla^{2}\right|_{\mathcal{P}_{t}}\right)
$$

where $\nabla$ is a $\mathcal{P}_{t}$-symplectic connection on the formal symplectic manifold $\left(M, \omega_{t}, \mathcal{P}_{t}\right)$.

Proof. Parts a) and b) follow from Proposition 8.5, Corollary 8.6, and Proposition 9.7 a). Part c) follows from Lemma 8.2. Part d) is the same as Proposition 9.7 b).

Remark 10.2. We have interpreted the form $\omega_{t}$ from (10.1) as a representative of the extension class associated to a PDQ (see Section 8). The form $\operatorname{tr}\left(\left.\nabla^{2}\right|_{\mathcal{P}_{t}}\right.$ ) is the curvature form of the connection induced by $\left.\nabla\right|_{\mathcal{P}_{t}}$ on the complex line bundle $\operatorname{det}\left(\mathcal{P}_{t}\right)$. Actually, $\operatorname{det}\left(\mathcal{P}_{t}\right)$ can be presented as a line $\mathcal{O}_{t}$-bundle, i.e. as a locally free sheaf of $\mathcal{O}_{t}$-modules of rank one. Indeed, $\operatorname{det}\left(\mathcal{P}_{t}\right)=\mathcal{C}_{M}^{\infty}[[t]] \otimes_{\mathcal{O}_{t}} \mathcal{L}$, where $\mathcal{L}=\Omega_{\mathcal{O}_{t}}^{n}, n=\frac{1}{2} \operatorname{dim} M$. The form $-\operatorname{tr}\left(\left.\nabla^{2}\right|_{\mathcal{P}_{t}}\right)$, as well as minus curvature form of any other connection on $\mathcal{L}$, can be interpreted as a representative of the extension class of an $\mathcal{O}_{t}$-extension of $\operatorname{Der}\left(\mathcal{O}_{t}\right)$ associated with $\mathcal{L}$. Indeed, let $\widetilde{T}_{\mathcal{L}}$ denote the sheaf of $\mathcal{O}_{t}$-differential operators on $\mathcal{L}$ of order at most one. Then $\widetilde{T}_{\mathcal{L}}$ equipped with the left $\mathcal{O}_{t}$-module structure and the Lie bracket given by the commutator naturally forms a $\mathcal{O}_{t}$-extension of $\operatorname{Der}\left(\mathcal{O}_{t}\right)$. Splittings of this extension are flat connection on $\mathcal{L}$. Let $d_{\alpha}$ be local flat connections on $\mathcal{L}$ in some open covering $\left\{U_{\alpha}\right\}$ of $M$. Then, $d_{\alpha}-d_{\beta}$ are closed 1-forms of $\Omega_{\mathcal{O}_{t}}^{1}$ that form a Čech cocycle. 
Hence, there exist smooth 1-forms $f_{\alpha} \in \mathcal{P}_{t}^{\perp}$ such that $d_{\alpha}-d_{\beta}=f_{\alpha}-f_{\beta}$. Differential operators $d_{\alpha}-f_{\alpha}$ form a global connection on $\mathcal{L}, \nabla_{\mathcal{L}}$, with the curvature locally equal to $-d f_{\alpha}$. On the other hand, by definition (see Section 8 ), the extension class of $\widetilde{T}_{\mathcal{L}}$ is represented by the form $d f_{\alpha} \in d \mathcal{P}_{t}^{\perp}$, i.e. $-\nabla_{\mathcal{L}}^{2}$. So, projecting the equality (10.1) to $\Gamma\left(M, d \mathcal{P}_{t}^{\perp}\right) / d\left(\Gamma\left(M, \mathcal{P}_{t}^{\perp}\right)\right)$, we obtain

$$
\left[\theta_{t}\right]=c l_{E x t}\left(\mu_{t}, \mathcal{O}_{t}\right)-\frac{t}{2} c l_{E x t}\left(\widetilde{T}_{\operatorname{det}\left(\mathcal{P}_{t}\right)}\right) .
$$

Details are left to the reader.

Note that the form $-\frac{1}{2 \pi \sqrt{-1}} \operatorname{tr}\left(\left.\nabla^{2}\right|_{\mathcal{P}_{t}}\right)$ represents the first Chern class of $\mathcal{P}$, [KN].

Corollary 10.3. Let $\mathbb{A}_{t}$ be a deformation quantization on $(M, \omega)$. Suppose its Fedosov class $c_{F}\left(\mathbb{A}_{t}\right)$ is represented by the form $\theta_{t}$ that has a polarization $\mathcal{P}_{t}$. Then $\mathbb{A}_{t}$ can be extended to a PDQ $\left(\mathbb{A}_{t}, \mathbb{O}_{t}\right)$, where $\mathbb{O}_{t}$ is isomorphic to $\mathcal{O}_{\mathcal{P}_{t}}$.

Proof. Let $\nabla$ be a $\mathcal{P}_{t}$-symplectic connection on the formal symplectic manifold $\left(M, \theta_{t}, \mathcal{P}_{t}\right)$. Let $\left(\mathcal{C}_{M}^{\infty}[[t]], \mu_{t}, \mathcal{O}_{t}\right)$ be a PSP such that

$$
\tau\left(\mathcal{C}_{M}^{\infty}[[t]], \mu_{t}, \mathcal{O}_{t}\right)=\left(\theta_{t}-\frac{t}{2} \operatorname{tr}\left(\left.\nabla^{2}\right|_{\mathcal{P}_{t}}\right), \mathcal{P}_{t}\right)
$$

By $(10.1), c l_{F}\left(\mathcal{C}_{M}^{\infty}[[t]], \mu_{t}\right)=\left[\theta_{t}\right]=c l_{F}\left(\mathbb{A}_{t}\right)$, therefore star-products $\mathbb{A}_{t}$ and $\left(\mathcal{C}_{M}^{\infty}[[t]], \mu_{t}\right)$ are equivalent.

Remark 10.4. All constructions of the paper can be extended to the case when $M$ is a formal manifold, $M_{\lambda}$, which is $M$ endowed with the function sheaf $C_{M}^{\infty}[[\lambda]], \lambda$ a formal parameter. A formal polarized symplectic manifold is a triple, $\left(M_{\lambda}, \omega_{\lambda}, \mathcal{P}_{\lambda}\right)$, where $\omega_{\lambda}$ is a formal symplectic form on $M_{\lambda}$ and $\mathcal{P}_{\lambda}$ its polarization. The above construction of a polarized star-product applied to a formal polarized symplectic manifold $\left(M_{\lambda}, \omega_{\lambda}, \mathcal{P}_{\lambda}\right)$ gives the following

Proposition 10.5. Let $\left(\mathbb{A}_{t}, \mathbb{O}_{t}\right)$ be a PDQ and $\left(\omega_{t}, \mathcal{P}_{t}\right)$ an element on the orbit corresponding to $\left(\mathbb{A}_{t}, \mathbb{O}_{t}\right)$. Then, there exists on $\left(M_{\lambda}, \omega_{\lambda}, \mathcal{P}_{\lambda}\right)$ a PSP

$$
\left(C_{M}^{\infty}[[\lambda]][[t]], \mu_{\lambda, t}, \mathcal{O}_{\lambda, t}\right)
$$

such that $\left(\mathbb{A}_{t}, \mathbb{O}_{t}\right)$ is equivalent to the diagonal sub-family $\left(C_{M}^{\infty}[[t]], \mu_{t, t}, \mathcal{O}_{t, t}\right)$ obtained by the substitution $\lambda=t$. 


\section{References}

[BFFLS] F. Bayen, M. Flato, C. Fronsdal, A. Lichnerowicz, and D. Sternheimer, Deformation Theory and Quantization, Ann. Physics 111 (1978), 61-110.

[BB] A. Beilinson and J. Bernstein, A proof of Jantzen conjectures. I. M. Gelfand Seminar, 1-50, Adv. Soviet Math., 16, Part 1, Amer. Math. Soc., Providence, RI, 1993.

[BK] A. Beilinson and D. Kazhdan, Geometric quantization, preprint.

[BD] P. Bressler and J. Donin, Polarized deformation quantization, math.QA/0007186.

[BW] M. Bordemann and S. Waldmann, A Fedosov star product of the Wick type for Kähler manifolds, Lett. Math. Phys. 41 (1997), no. 3, 243-253.

[De] P. Deligne, Dèformations de l'Algébre def Fonctions d'une Variété Symplectique: Comparaison entre Fedosov et De Wilde, Lecomte, Selecta Mathematica, New Series, v. 1, n. 4 (1995), 667-697.

[Fe] B. Fedosov, "Deformation Quantization and Index Theory", Academie Verlag, v. 9.

[Ka1] A. Karabegov, Deformation Quantization with Separation of Variables on Kähler Manifold, Commun. Math. Phys., v. 180 (1996), 745-755.

[Ka2] A. Karabegov, Cohomological Classification of Deformation Quantizations with Separation of Variables, Lett. Math. Phys., v. 43 (1998), 347-357.

[KS] A. Karabegov and M. Schlichenmaier, Almost Käler Deformation Quantization, math.QA/0102169.

[KN] S. Kobayashi and K. Nomizu, "Foundations of Differential Geometry, Volume II", Interscience Publishers, 1969.

[Ko] M. Kontsevich, Deformation quantization of Poisson manifolds, I, q-alg/9709040.

[NT] R. Nest and B. Tsygan, Algebraic Index Theorem for Families, Adv. Math. 113 (1995), 151-205.

[Ne] N. Neumaier, Universality of Fedosov's Construction for Star Products of Wick Type on Semi-Käler Manifolds, math.QA/0204031.

[Ra] J. H. Rawnsley, Polarization and diagonal quantization, Trans. AMS, 230, 1977, 235-255.

[RY] N. Reshetikhin and M. Yakimov, Deformation Quantization of Lagrangean Fiber Bundles, preprint, math/9907164.

[Xu] P. Xu, Fedosov's $\star$-products and quantum momentum maps, Comm. Math. Phys., 197 (1998), 167-197.

e-mail: donin@macs.biu.ac.il 